\title{
ZUR LINGUISTIK DER METAPHER ${ }^{1}$
}

\section{Von Werner Abrabam}

0. Beim täglichen Gebrauch der Sprache kommen "unwörtliche" Bedeutungen wie in Fuß des Klaviers, Hals der Flasche, Kopf des Bettes, Stirn des Kastens, Bein des Tisches, Flanke des Berges andauernd vor.

Dies mögen einigermaßen gefestigte, also lexikalisierte Verwendungen von Fuß, Hals, Kopf, Stirn, Bein, Flanke sein. Daneben - und wie oft eigentlich? - sind wir bei der Verwendung von übertragenen Bedeutungen durchaus innovativ: statt Er schlingerte in das Zimmer wie ein stenerloses Schiff im wild bewegten Meer sagen wir einfach Er schlingerte in das Zimmer, statt Das Mädchen war verwelkt wie die Blütenblätter einer Blume sagen wir einfach Das Mädchen war verwelkt. Die Forderung, daß solch metaphorischer Gebrauch von Lexemen oder Lexemverbindungen im Sinne einer generativen Grammatik im Lexikon einfach eine eigene Beschreibung erhalten müsse, ist deswegen zweifellos falsch, weil zu simplistisch. Dies kann jedenfalls nur für Metaphernklischees ("frozen metaphors") gelten, und auch die Angabe von entsprechenden Regeln der Bedeutungsrelation zwischen wörtlichem Gebrauch eines Ausdrucks und metaphorischem Gebrauch darf nur dort wegfallen, wo er ohnedies nicht herstellbar ist, d. h. wo die Relation zwischen metaphorischem Gebrauch einer Äußerung und dem wörtlichen Gebrauch die zwischen definiendum und definiens ist, ohne daß die Regeln der Bedeutungsübertragung und die Bedingungen, unter denen diese Bedeutungsiibertragung elizitiert wird, explizit gemacht werden müssen.

Ich nehme bei meinen Ưberlegungen aufs erste einen exegetisch-terminologisch naiven Standpunkt zu dem Begriff „Metapher" ein. Ich gehe auf keine Definition des Begriffs zurück - erstens weil es eine Menge von nicht übereinstimmenden definitorischen Versuchen gibt und zweitens weil ich für die Erfüllung der alten Harwegschen Forderung, Meta-

${ }^{1}$ Eine erste Version dieses Aufsatzes konnte ich durch eine Reihe von Sitzungen hindurch mit Graham Stuart, G. J. Luhrmann, J. de Mey und Eric Reuland, alle vom Instituut voor Algemene Taalwetenschap der Rijksuniversiteit Groningen, durchdiskutieren. Ihnen allen danke ich wertvolle Kritik, im besonderen aber J. de Mey und E. Reuland, die mich vor manchem Fehler bewahrt haben. Die entscheidende Anregung zu den folgenden Überlegungen geht auf die Lektüre von Kintsch 1972 (1971) zurück. Eine beträchtlich erweiterte englische Fassung ist abgedruckt in Abraham 1975. 
pher nach dem zu erwartenden Gewinn einer Begriffserklärung zu definieren, kein Kriterium besitze.

"Metapher" verwende ich vielmehr im Sinne von „Bedeutungsübertragung" und schränke diesen Oberbegriff, der ja auch Allegorie, Gleichnis, Symbol u. a. umfaßt, im wesentlichen ein durch Verwendung von Wörtern in Sätzen oder Satzteilen im nicht-wörtlichen Sinne. ${ }^{2}$ Explizit sehe ich mich damit in der Nähe der Erscheinungen die traditionell mit Metonymie, Katachrese, Synekdoche u.e.a. klassifiziert werden. Ich sehe mich auch in unmittelbarer Nähe des Begriffs der Semigrammatikalität, wie er von Chomsky geprägt wurde. So ist der Satz

\section{Green ideas sleep furiously}

einen bestimmten Kontext vorausgesetzt, durchaus sinnvoll: dann nämlich, wenn dieser Kontext es gestattet, die Verletzungen der Selektionsregeln durch "Personifikation von ideas", die Zuordnung von „Materialität und damit die Eigenschaft, Farbe zu tragen, an ideas" sowie die Zuweisung des Charakteristikums "aktiv“ an sleep aufzulösen. Vorausgesetzt daß unsere Welterfahrung einen kompatiblen Kontext dazu findet, ist jede Verletzung auf der Ebene der Selektionsregeln, also den "semantischsten" unter den grammatischen Regeln, als Metapher zu interpretieren. Diese Konsequenz gilt nicht umgekehrt, d. h. nicht jede Metapher ist durch eine Verletzung von Selektionsregeln zu erklären. Die Sinne von Der Mensch ist ein Wolf oder Die Schwarzen sind die Armen von Europa sind nicht mithilfe von solchen Regelverstößen zu interpretieren. So wie für das erste Chomskysche Beispiel gilt jedoch für alle metaphorischen Sinne von Sätzen, daß sie durch syntaktische Wohlgeformtheit gekennzeichnet sind. Dies läßt sich anhand eines extremen Beispiels wie de-de-de erklären: Erst wenn wir z. B. die nur auf syntaktischen Kategorien und deren Relationen zueinander aufbauende Struktur (1) zuweisen können, ist Interpretabilität gegeben - vorausgesetzt ein Kontext liegt vor, aus dem die Zuweisung von Nominal-, Artikel- und Verbalkategorie an de plausibel wird.

Mit dieser Erklärung möchte ich auch das Kapitel der „grammatischen Metapher", das viele Leute beschäftigt hat, umreißen und im wesent-

${ }^{2}$ Hiermit schließe ich jenen „Metaphertyp“ aus, der über die Satzgrenze hinaus sich in Textteilen dadurch manifestiert, daß durch bestimmte lexikalische Elemente Themaerwartungen etabliert, aber dann enttäuscht werden. Zu diesem Begriff der "Textmetapher" und den Folgerungen für ein Instrumentarium der konsistenten Beschreibung solcher Metaphern vgl. vor allem Siebert 1973. 
(1)

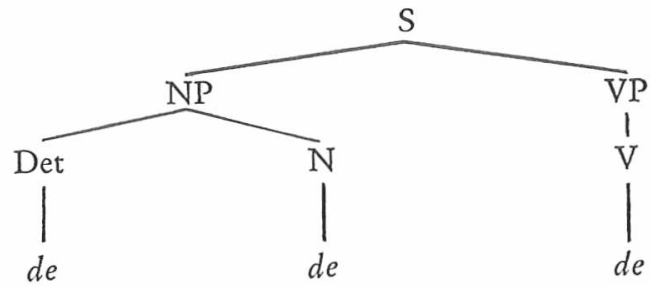

lichen erledigen. $D u r u f t$ ist einfach ein nicht angezeigter Wechsel von formaler zu materialer Supposition, sofort verständlich $\mathrm{zu}$ machen durch „Du" ruft und jedenfalls syntaktisch darstellbar durch (2).

(2)

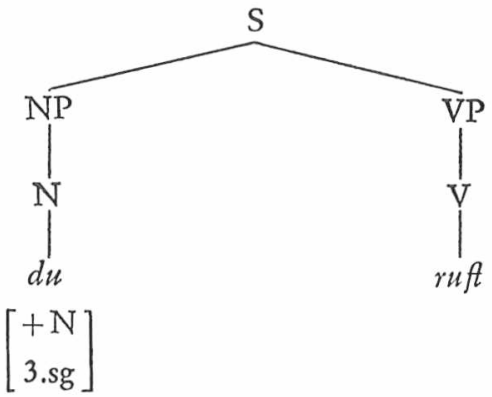

Ich möchte meine folgenden Bemerkungen und Überlegungen in 5 Hauptabschnitte einteilen:

1. Eine kurze Diskussion der pragmatischen Bedingungen, die erfüllt sein müssen, wenn man einer Struktur das Prädikat "Metapher" zuweist.

2. Bedingungen für den Gebrauch von Sprache in metaphorischer Funktion: eine Skizze der Forschung.

3. Substitutionsthese - Vergleichsthese - Interaktionsthese

4. Bemerkungen zur Rolle der Begriffe „wörtliche Bedeutung" und "Akzeptabilität" bei der Erklärung von Metaphern: Priorisierung, Akzeptabilitätsgrade und alternative Welten.

5. Einige praktische Lösungsfälle. 
1. Der folgende terminologische Rabmen wird in der Folge benutzt (Nach Black: 47, aber unter Änderung dessen Terminologie):

(1) E ... expression, Ausdruck, Äußerung

(2) F ... verbal „frame", Kontext

(3) $F(E)$ heißt dann „E mit inkompatiblem Kontext F“

(4) Weiteres sei: $m$ '(E) ... Bedeutung von $E$ in $F(E)$

(5) $m(X) \ldots$ Bedeutung von $X$ wobei gilt: 5.1. $\mathrm{m}^{\prime}(\mathrm{E})=\mathrm{m}(\mathrm{X})$, so daß $\mathrm{X}$ das Substituens für $E$ in $F(E)$ und $E$ in $F(E)$ das Substituendum.

5.2. $\mathrm{m}^{\prime}(\ldots)=$ metaphorischer Gebrauch (= nichtwörtliche u. a. Charakteristika), also: übertragene Bedeutung;

5.3. $\mathrm{m}(\ldots)=$ wörtlicher Gebrauch

Es ist offenbar interessant zweierlei zu untersuchen:

(6) es gilt, die linguistischen Mittel zu finden, um die Auswirkung von $\mathrm{F}$ auf $\mathrm{E}$ derart, daß $\mathrm{E}$ als sinnvoll (im Sinne eines $\mathrm{X}$ ) interpretiert wird, zu beschreiben.

(7) es gilt die Grenzen und Bedingungen dafür zu finden, daß E in inkompatiblem Kontext interpretierbar wird.

(6) ist eine Frage der Linguistik und Logik (als strenger Beschreibungssysteme);

(7) ist eine Frage der Philosophie und der sozialen Wissenschaften, daneben der Pragmatik als einer der Wissenschaften, die die Form dieser Diskussion bestimmt.

Unter den Kategorien, die die Pragmatik einbringt, sind $S p r$ und $A d r$. Wenn wir von der nicht auszuschließenden Annahme ausgehen, daß die Bedeutungen eines Teils der von Spr und Adr verwendeten Wörter $\mathrm{n}$ ich $\mathrm{t}$ identisch sind, dann kommt zur Begriffsbildung oben noch dazu:

(8) $\mathrm{m}_{\mathrm{sp}}(\mathrm{X})$ $m_{\text {Adr }}(X)$

Die Spr und Adr gemeinsame Bedeutung von $\mathrm{X}$ sei repräsentiert durch (9) $\mathrm{R}\left[\mathrm{m}_{\mathrm{sp}}(\mathrm{X}), \mathrm{m}_{\mathrm{Adr}}(\mathrm{X})\right]$

wobei $\mathrm{R}$ den Durchschnitt an verifizierenden Bedingungen (semantischen Merkmalen) für den Gebrauch von X bezeichnet. Für $\mathrm{R}$ gibt es folgende prinzipielle Möglichkeiten:

(10) $\mathrm{R}=\phi \quad$ d. h. $\quad\left\{\mathrm{m}_{\mathrm{sp}}(\mathrm{X})\right\} \cap\left\{\mathrm{m}_{\text {Adr }}(\mathrm{X})\right\}=\varnothing$

$\mathrm{R}=$ Identität (im Idealfall) 
Dazwischen gibt es Abstufungsgrade von Durchschnitten von Bedeutungsmerkmalen; diese Grade sind wesentlich für eine Problematisierung von Norm einerseits und Interpretabilität (Akzeptabilität) andererseits.

Es muß deutlich sein, daß mit dieser pragmatischen Diskussion des Substituens für das metaphorisch gebrauchte Lexem auch die wörtliche Bedeutung von $\mathrm{E}$ außerhalb von $\mathrm{F}$ erfaßt wird, d. h. daß dasselbe für $\mathrm{E}$ dort gilt, wo $m(E) \neq m(X)$. Um unter allen $F(E)$ scheiden zu können zwischen (mehr oder weniger akzeptablen) Metaphern und nichtsinnvollen (nicht-interpretierbaren) inkompatiblen Strukturen, gehen wir auf einige der Gricesche Diskursimplikaturen ein (nach Apostel: 13 ff.):

(11) Spr weiß, daß Adr E eine Bedeutung zuordnet, die sich nicht zu sehr von der des Spr unterscheidet: $\mathrm{m}_{\mathrm{sp}}(\mathrm{X}) \cong \mathrm{m}_{\mathrm{Adr}}(\mathrm{X})$.

(12) Spr intendiert, daß Adr diese Bedeutungszuordnung vornimmt.

(13) Adr weiß, daß es Spr war, der E äußerte, welches Adr wahrgenommen hat.

(14) Spr weiß (oder glaubt), daß Adr, wenn er E wahrnimmt, weiß (oder glaubt), daß Spr m(E) kennt (oder zu kennen glaubt).

(15) Wenn Adr E wahrnimmt, dann weiß (glaubt) Adr, daß Spr m(E) weiß (oder glaubt zu wissen).

Folgender Zusammenhang besteht $\mathrm{z}$ wischen (14) und (15):

15.1. Wiss $(x, m) \supset \mathrm{m}$

15.2. Glaub $(x, m) D m$

wobei $m$ bedeutet die Anwendungsbedingungen (die die richtige Anwendung garantierenden Charakteristiken [= Merkmale]) für das Zeichen E; d. h. (15) ist nicht nötig, wenn in (14) nur der epistemische Operator Wiss verwendet wird.

Bedingung (15) schließt Lüge, Prätention von Spr sowie allgemeines Mißverständnis von Adr aus.

(16) Spr intendiert, daß Adr weiß/glaubt, daß Spr m(E) kennt/glaubt zu kennen.

(17) Die Wahrnehmung von E erzeugt genau diesen Erkenntniszustand bei Adr über Spr; denn Adr glaubt, daß E zu einer Menge $\{E\}$ mit einer solchen strukturellen Beschreibung gehört, daß nur Systeme, die in Übereinstimmung sind mit den Regeln einer bestimmten Sprache, Elemente von $\{E\}$ erzeugen. Adr glaubt, daß die Regeln dieser Sprache so beschaffen sind, daß nur die Kenntnis von $m(E)$ und die Intention, $\mathrm{da} ß$ andere ebenfalls $\mathrm{m}(\mathrm{E})$ kennen, ein solches $\mathrm{E}$ produzieren. 
(18) Normalerweise gilt (ist wahr), (a) daß Spr in Übereinkunft mit diesen Regeln der Sprache, R, handelt, und (b) daß Spr glaubt, daß Adr glaubt, daß Spr m(E) zu kennen glaubt.

Bei Nichtgültigkeit dieser Bedingungen, (a) und (b), gibt es prinzipiell zwei Möglichkeiten:

18.1. Mißverständnis, z. B. wenn (a) verletzt ist, oder wenn Adr glaubt, daß Spr m(E) glaubt (weiß), während Spr will, daß Adr glaubt, daß Spr $\mathrm{m}^{\prime \prime}(\mathrm{E})$ glaubt (weiß), wobei $\mathrm{m}(\mathrm{E}) \neq \mathrm{m}^{\prime \prime}(\mathrm{E})$. Dies gilt auch für den Ungleichheitsfall, wo $m(E) \cap m^{\prime \prime}(E)$ gleich ist entweder $\mathrm{m}(\mathrm{E})$ oder aber $\mathrm{m}^{\prime \prime}(\mathrm{E})$, also in einem Enthaltenseinverhältnis oder Implikationsverhältnis.

18.2. Nichtmißverständnis (a) wenn Adr die Abweichung von $R$ erkennt (erwartet), oder (b) wenn Spr glaubt (weiß), daß Adr glaubt (weiß), daß Spr nicht $\mathrm{m}(\mathrm{E})$, sondern $\mathrm{m}^{\prime}(\mathrm{E})$ oder $\mathrm{m}^{\prime \prime}(\mathrm{E})$ glaubt (weiß).

(15) und (18) sind Bedingungen, die „ehrliches Reden" beschreiben, d.h. die Intention von Spr, daß Adr ihn so versteht, wie Spr es intendiert hat und wie es die Spr und Adr gleichermaßen vertrauten Regeln der Sprache garantieren. Dies ist jedoch eine Idealisierung, da es $u$. a. eine Sprachnorm für $\mathrm{Spr}$ und $\mathrm{Adr}$ voraussetzt (vgl. oben: $\mathrm{m}_{\mathrm{sp}}(\mathrm{X})$ $\cong \mathrm{m}_{\mathrm{Adr}}(\mathrm{X})$ ); zudem schließt diese Einschränkung wie gesagt Sprechhaltungen wie 'Täuschung, Prätention (Spr will, daß Adr glaubt, daß Spr $\mathrm{m}(\mathrm{X})$ glaubt (weiß), während Spr in Wirklichkeit $\mathrm{m}^{\prime \prime}(\mathrm{X})$ glaubt [weiß]) oder Unsinnreden, Verwirrung des Spr, (Spr weiß nicht, daß Adr nicht weiß, daß Spr m(E) weiß - Ignoranz von Spr über die Normalgradigkeit von Spr $[\mathrm{m}(\mathrm{E})]$ - oder Spr kennt überhaupt keine Anwendungsregel für $\mathrm{E}, \mathrm{d}$. h. er verwendet es sinnlos) und schließlich Manipulation des Verständnisses (Spr will nicht, daß Spr m(E) weiß [glaubt]) aus.

Irritation und Dissoziation vom Normalverständnis - Haltungen, die wir in der modernen Literatur antreffen - müssen mit dieser zweiten Annahme erklärt werden; metaphorischer Gebrauch setzt die Intention des Spr (Metapherngebrauchers, Metaphernproduzenten) voraus, daß Adr versteht, was Spr will - dies gilt jedenfalls bei jenem Typus von Sprachverwendung, bei dem Spr einen Rezipienten und dessen mögliche Erwartungen reflektiert, jedenfalls also im Diskurs, im Brief, im Vortrag usw. Bleibt das Verständnis von $\mathrm{F}(\mathrm{E})$ bei Adr aus, so wird sich Adr über $m_{\mathrm{sp}}(\mathrm{E})$ bzw. über jene Implikationen, die eine Analogie zwischen $m(X)$ und $m^{\prime}(E)$ ermöglichen, durch Rückfrage vergewissern. 
Der metaphorische Sprachgebrauch im Dialog/Diskurs ist also gewöhnlich von anderen Strategien bei Adr geprägt als im Falle jenes metaphorischen Sprachgebrauchs, wo diese Rückversicherung nicht möglich ist, also im Geschriebenen. Hier ist Adr auf den Aufbau der nötigen Implikationen selbst angewiesen, um das Substituens, $m(X)$, zu finden; er hat weiter die wesentliche Aufgabe herauszufinden, ob $m(X)$ überhaupt als Substituens von Spr intendiert war.

Wie Adr die letzte Frage entscheidet, richtet sich neben der Beziehung zwischen den alternativen Welten von Spr und Adr auch danach, ob die folgende Diskursprämisse gilt oder nicht: Ob Adr weiß/glaubt/daran zweifelt, daß Spr mit E überhaupt etwas meint. Diese Prämisse lautet verallgemeinert so - sie unterscheidet sich von (15) dadurch, daß sie noch allgemeiner ist.

(19) Adr weiß (glaubt), daß Spr mit E einen Erkenntniszustand derart schaffen will, daß Adr glaubt, daß Spr m(E) weiß (glaubt, wissen). Die Entscheidung von (19) richtet sich nach den Erwartungen, die Adr über die Glaubwürdigkeit von Spr besitzt.

Metaphorischer Sprachgebrauch des Spr setzt per definitionem diese Diskursimplikation (19) voraus. Sie ist der primäre Erkenntniszustand, der vorliegen muß, bevor Adr an Interpretationsversuche von semantisch inkompatiblen, aber grammatisch wohlgeformten Strukturen herangeht. (19) schafft, will Adr einer zunächst noch nicht interpretierten Struktur das Prädikat „metaphorischer Gebrauch" zuteilen, auch die Voraussetzung, daß Spr die Analogie zwischen $m^{\prime}(E)$ und $m(X)$ bewußt hergestellt hat - damit sind bildliche Kindersprache, Katachrese, abweichender Wortgebrauch bei Aphasikern usw. nicht als (mögliche) Metaphern zu verstehen, sondern es sind sprachliche Konventionen, bei denen zur Funktion von $\mathrm{F}(\mathrm{E})$, also $\mathrm{m}^{\prime}(\mathrm{E})$, kein Analogon $\mathrm{m}(\mathrm{X})$ zugrundegelegt (mitgedacht) wird.

\section{Bedingungen für den Gebrauch von Sprache in metaphorischer Funktion: eine Skizze der Forschung}

Während die Literatur zur Metapher sich hauptsächlich mit der Frage beschäftigt hat „Was ist eine Metapher?" bzw. „Was tut eine Metapher?", greift z. B. Helmer (1972) die Frage auf, die vor allem in der psychologischen Literatur gestellt worden ist: „Unter welchen Bedingungen kann eine Person eine Metapher benützen?" Die Antwort auf 
die erste Frage, die in diesem Zusammenhang auch immer wieder zitiert wird, wird versucht von Vertretern der Philosophie und Kunstkritik (Goodman [1968] und Black [1962]). Metapher ist hier eine Sache der Bedeutung von Wörtern oder Wortgruppen, d. h. eine Frage der Beziehung von Symbolen oder Symbolschemata zu einem referierten $\mathrm{Ob}-$ jekt bzw. einer Menge von solchen. Eine Antwort auf die zweite Frage versucht eine umfangmäßig weit geringere psychologische Literatur (vgl. Helmer, 5). Dieser Literatur geht es darum, Metapher in Abhängigkeit von bestimmten Haltungen, Erfahrungen oder Dispositionen bzw. als Komplex von Reaktionen beim Sprecher und Adressaten zu beschreiben.

Wiewohl ich im folgenden hauptsächlich - aber nicht ausschließlich Vorschläge aufgreifen und diskutieren möchte, die sich mit der ersten Frage beschäftigen, scheint es mir doch wertvoll, kurz auf einige Punkte zum zweiten Fragenkomplex einzugehen. Dabei ist besonders ein Fragenkreis einigermaßen untersucht worden: nämlich derjenige, der sich an die Frage anschließt, welche Gruppe von Leuten offenbar mehr oder weniger unfähig ist, Metaphern zu gebrauchen oder zu verstehen. Jakobson und Halle (1956) haben unter anderem den Typus von Aphasie beschrieben, in dem der Aphasiker Wörter bei Verwendung in ihrer wörtlichen Bedeutung versteht, nicht aber bei Verwendung in einer metaphorischen Bedeutung. Da die Bedeutung eines Wortes mitbestimmt wird von dem Kontext, in dem dieses Wort gebraucht wird (dem sprachlichen Kontext), in dem es erscheint, und dem situativen Kontext, auf den es sich bezieht), muß demnach die Unfähigkeit, Metaphern richtig zu interpretieren, damit zusammenhängen, daß der Kontext für den Aphasiker jeweils nur eine einzige Wortbedeutung freimacht. Ist dieser bestimmte eine spezifische Bedeutung eines Zeichens freimachende Kontext nicht vorhanden, dann kann der Aphasiker den Satz oder die Wortgruppe nicht produzieren. Die Ähnlichkeiten zwischen ein und demselben Wort, in verschiedenen Kontexten verwendet, um damit verschiedene Objekte zu kennzeichnen oder zwischen verschiedenen Wörtern, die in ähnlichen Kontexten verwendet werden, um ähnliche Objekte zu kennzeichnen, bleiben ihm verborgen. Jakobson (1960: 356; nach Helmer 1972: 9) charakterisiert diese Unfähigkeit in seiner allgemeinen Form als Verlust der Fähigkeit für metasprachliche Operationen: der Verlust also der Fähigkeit, Wörter zu verwenden, die sich auf andere Wörter beziehen oder andere Wörter kennzeichnen. 
Ähnliche sprachliche Fehlleistungen bei der Unterscheidung von metaphorischem von wörtlichem Sprachgebrauch werden berichtigt für Schizophreniker und akute Suizidfälle. Im zweiten Fall handelt es sich natürlich nicht um pathologische Unfähigkeit, sondern um eine Kommunikationsform, die unter der Motivation eines suiziden Zustandes nicht zu dem stilistischen Mittel der Metapher greift (Osgood 1960). Kinder bis zum Alter von 11 bis 12 verwenden Wörter gewöhnlich gleichfalls nicht metaphorisch, es sei denn in oft gebrauchten Klischees. Falls solche klischierten "Wortübertragungen" vom Kind aber wiederverwendet werden, so wird kein Zusammenhang zwischen diesen beiden Verwendungen erkannt: d. h. es wird keine Regel zur Bedeutungsübertragung wirksam, die in Verwendung geschieht vielmehr aufgrund einer isolierten Speicherung im Gedächtnis (im Lexikon). So wird unter Umständen hart sowohl auf feste Objekte wie auf den Vater eines Freundes des Versuchskindes verwendet, das Kind assoziiert aber diese beiden Wortverwendungen nicht miteinander (Asch/Nerlove [1960]). Die Extensionen dieser beiden Bedeutungen, so können wir sagen, haben keine Gemeinsamkeiten, so daß eine Bedeutungsähnlichkeit nicht hergestellt werden kann.

Diesen Beobachtungen widersprechen Cohen/Margalit (1970: 470): "Children do not learn to speak metaphorically as a kind of crowning achievement in the apprenticeship of language-learning. Rather they use metaphors naturally from infancy onwards, and have gradually to learn - with respect to each noun, verb, adjective or adverb - how to speak literally." Der Widerspruch ist aber aufzulösen durch den jeweils verschiedenen Metaphernbegriff: Metapher ist der Geschichte des Terminus nach (so wie Symbol, Allegorie, Gleichnis - Begriffe, die Cohen/Margalit mit Metapher auf einer Ebene nennen) auf die bew u ß te Verwendung einer Bedeutungsübertragung hin festgelegt. Dieses implizite Verständnis liegt übrigens auch in Cohen/Margalits eigener Gegenüberstellung zur wörtlichen Verwendung: Was kann „metaphorisch" anderes sein als das bewußte Ändern des "wörtlichen" Zeichengebrauchs? Denn wenn das Kind the car shouts (statt boots) sagt, so handelt es sich um den w örtlich en Gebrauch von shout beim Kind - es sei denn das Kind verwendet shout gegen sein besseres Wissen, weil ihm das richtige hoot augenblicklich nicht einfällt. Metaphernproduktion setzt voraus, daß man jedenfalls die Wahl der wörtlichen Verwendung hat und die Abweichung der metaphorischen Verwendung von dieser wörtlichen stilistisch ausmißt. „Wörtliche Bedeutung" 
setzt die Konstruktion von normaler Bedeutung voraus (vgl. die Definition S. 94), eines Begriffs den Cohen/Margalit nicht eingeführt haben und der hier entscheidend ist.

Helmers Absicht in seiner kurzen Darstellung zum zweiten Fragekomplex ist es, auf die Parallele zwischen "metaphorischer Inkompetenz" und „metasprachlicher Inkompetenz" hinzuweisen. Das ist nichts anderes als die Diagnose über die Nichtfähigkeit, Wörter zu gebrauchen, um auf andere Wörter hinzuweisen, andere Wörter zu beschreiben oder zu kennzeichnen. Dies impliziert deutlich, daß Metapher aufgefaßt wird als Prozeß, in dem nach bestimmten Regularitäten die Bedeutung eines bestimmten Wortes durch ein anderes Wort vermittelt wird. Dies wird in der Literatur zur Frage 1 gewöhnlich als Substitutionsthese oder als Vergleichsthese dargestellt.

\section{Substitutionsthese - Vergleichsthese - Interaktionsthese}

3.1. Welche Definitionen von „Metapher" man auch hernimmt, d. h. so sehr diese in Details auch von einander abweichen, so ist ihnen doch eines gemeinsam: nämlich daß es sich dabei um eine Bedeutungsübertragung handelt. In diesem Sinne beschreiben die Prozesse der Metonymie, der Synekdoche (pars pro toto-Bildung), Gleichnis, Bild (imagery), Allegorie usw. in oft nicht klar gegen einander abgrenzbarer Weise ähnliche Formen der Bedeutungsübertragung. Ich möchte hier nicht auf die Definitionen von Metapher eingehen; es scheint mir auch nicht die zentrale Frage zu sein (vielleicht deswegen, weil ich die Exegese der Begriffsbildung in der Sekundärliteratur uninteressant finde). Ich möchte vielmehr zwei Interpretationen von Black (1962) zum Komplex der Metapher übernehmen und nach (mikro-)linguistischen Aspekten hin diskutieren. Ich meine, daß die grundsätzliche Klassifizierung von Black den wesentlichen Teil aller Prozesse der Bedeutungsübertragung deckt, so daß ich mich nicht eigentlich auf die Metapher zu beschränken brauchte. Sie ist nur Ausgangspunkt der Diskussion.

Einige Beispiele zu der Behauptung, daß ein Substituens in wörtlicher Bedeutung, $\mathrm{m}(\mathrm{X})$, zum Verständnis der Bedeutungsübertragung nötig ist.

(1) Er fraß sich durch den Berg von Korrespondenz hindurch.

(2) Er posaunte die Nachricht binaus.

(3) Der Regen trommelte auf das Dach.

(4) Der Mensch ist ein Wolf. 
(5) Die Armen sind die Schwarzen Europas.

(6) Light is but the shadow of God.

In den ersten drei Beispielen kann jeweils ein Synonym oder eine bedeutungsgleiche Wortgruppe eingesetzt werden, die die wörtliche Bedeutung des Satzes herstellt: Die Prädikate sind in den Sätzen (1)-(3) jeweils zu ersetzen durch arbeiten; jedem, der es hören will, mitteilen; in kurzen und regelmäßigen Abständen schlagen. Solche Fälle von übertragender Bedeutung deckt die Substitutionsthese.

In diesen drei ersten Fällen kann offenbar auch eine WIE-Relation hergestellt werden: das Schlagen des Regens auf das Dach ist WIE das Trommeln des Regens auf das Dach. Es ist interessant, daß in den beiden anderen Beispielen die WIE-Relation offenbar in Verbindung mit Topoi aus unserem enzyklopädischen Wissen elizitiert werden:Die WIERelation zwischen binausposaunen und die Nachricht verbreiten, so daß sie jedermann bört ist assoziiert mit dem Bild des Königsherolds bzw. des Nomenklators, die Ähnlichkeitsrelation zwischen sich durcharbeiten (oder sich durchwüblen) und sich durchfressen an den Topos vom Grießberg im Schlaraffenland. Dies scheint mir allerdings für die Erklärung des Zustandekommens der übertragenen Bedeutung in diesen Fällen nicht notwendig zu sein. D. h. die Metapherinterpretation könnte auch ohne die Assoziation mit diesen Topoi zustandekommen. Ich möchte diese Frage im Zusammenhang mit dem Komplex „Akzeptabilität" und "Lexikonzustand" noch einmal aufgreifen. Deutlich muß nur sein, daß sämtliche Fälle, die mithilfe von Substitution erklärt werden können, auch durch die Vergleichsthese gelöst werden können. Black (1962: $38 \mathrm{ff}$.) postuliert eine eigene Klasse von Metaphern, die mittels der Substitutionsprobe oder der Vergleichsprobe nicht erklärt werden können. Fälle wie (5): Die Armen sind die Schwarzen von Europa seien nicht so lösbar, daß man ein bestimmtes Wort oder eine Wortkette für Elemente in (5) einsetzt. Vielmehr, glaubt Black (5) so erklären zu müssen, daß die "Gedanken" über die Armen Europas und die amerikanischen Schwarzen in einer solchen "Interaktionsform" zueinander stehen, daß sie eine gemeinsame Bedeutung, eben die in (5) vermittelte, ergeben.

Ich werde im folgenden versuchen darzulegen, daß diese Beobachtung alleine nicht ausreicht, um eine spezifische Klasse von Metaphernbildung zu konstituieren. Vielmehr möchte ich das Phänomen der Metaphernproduktion und Metapherninterpretation allgemein mithilfe der Vergleichsthese erklären. Die Beobachtungen von Richards und Black zur 
Interaktion der an einem metaphorischen Ausdruck beteiligten lexikalischen Elemente ist jedoch ein wichtiger Ansatz zur linguistischen Erklärung des metaphorischen Prozesses. Für das Beispiel (5) ist demnach wie für die anderen Beispiele eine WIE-Relation herzustellen etwa der Form "Die Armen Europas sind wie die Schwarzen von Amerika". Damit ist aber bis auf ein Postulat über generelle formale Übertragungsprozesse hinaus noch nicht erklärt, wie es zu dem Vergleich zwischen den Armen Europas und den Schwarzen Amerikas kommen darf. D. h. wir müssen Regularitäten finden, die in der Semantik (oder Pragmatik bzw. Enzyklopädie) der Begriffe die Schwarzen Amerikas und die Armen Europas zusammengefaßt sind, und zwar der Art, daß ein Vergleich anderer arbiträrer Elemente ausgeschlossen wird, d. h. daß dann eine Metapherninterpretation nicht gelingt.

\section{2. "Fokus" und „Kontext"}

Metaphern entstehen uneingeschränkt durch Verletzungen der Kompatibilität zwischen Lexemen in syntaktischer Verknüpfung. Syntaktische Regularitäten (Konstituentenstrukturregeln) der Basis oder abgeleiteter Natur) bleiben jedoch vollkommen unangetastet. In einer Abweichungsgrammatik - die auch Verletzungen syntaktischer Natur Interpretationen zuteilt (vgl. Abraham 1971; Abraham/Braunmüller 1971) - werden demnach die Regeltypen der strengen Subkategorisierung und der Selektion eindeutig trennen zwischen syntaktischer Nichtinterpretierbarkeit (eventuell wohl aber semantischer, vgl. Abraham/ Braunmüller 1971) einerseits und metaphorischer Interpretierbarkeit andererseits.

Die verborientierten Selektionsbeschränkungen sind echt enthalten in der Menge der Kompatibilitätsregeln. Ihnen wurde allerdings nie eine kognitive Interpretation gegeben. Trotzdem müssen die Verstöße gegen die Selektionsbeschränkungen einer bestimmten Einschränkung unterliegen, um sinnlose Sätze auszuschließen. Man betrachte zum Beispiel Satz (7)

(7) In der Nacht sind alle Katzen schwarz.

Es geht um die Verträglichkeit von Katze, bzw. von dessen Quantifikation mit alle, mit deren Prädikaten mit sind schwarz. Dies ist offenbar im Widerspruch zu unserer Erfahrung. Denn wenn unter den Merkmalen einer Katze - komponentialanalytisch konzipiert - auch solche wie (Weiß) v (Braun) v (Gefleckt) und nicht nur (Schwarz) sind, dann widerspricht dies der All-Aussage über dieser Merkmalvereinigung. 
Dieser Widerspruch in alle Katzen sind schwarz fordert eine Auflösung - es sei denn man nimmt ihn als Sinnlosigkeit, als Nichtzuverstehendes hin. Offenbar geschieht letzteres auch, sofern nicht der Sprecher einer solchen Äußerung einen bestimmten Erwartungshorizont beim Beurteiler konstituiert, so daß dieser nach dem „tieferen Sinn", dem „nichtwörtlichen Sinn" sucht, der gemeint sein könnte und der in einem bestimmten Redekontext kompatibel und informationsbringend sein könnte. In diesem konkreten Fall ist es der Rückgriff auf den Kontext des Satzadverbials in der Nacht, mit dem der Widerspruch auflösbar ist. Der Vorgang hat etwas mit der phonologischen Neutralisation gemeinsam: In dem merkmalanalytisch erfaßten Kontext von in der Nacht [selbst markiert mit (Dunkel), (Farblos), (Schwarz)] wird die Disjunktion der Farbcharakteristik (Weiß) v (Braun) v (Gefleckt), kurz „Komplement von (Schwarz)“, neutralisiert zu (Dunkel) bzw. (Farblos), was mit (Schwarz) kompatibel ist.

(8) $[[($ Weiß $) \vee($ Braun $) \vee($ Gefleckt $) \vee \ldots \vee($ Schwarz $)] /$ $/$ (Katze) $]$ im Kontext [(Schwarz)/ (Nacht) $]$ $\Rightarrow[($ Schwarz $) /$ (Katze) $]$ im Kontext [(Schwarz)/(Nacht) $]$ Voraussetzung ist dabei das Akzeptieren der Eigenschaftszuordnung ist schwarz als von den Lichtverhältnissen abhängig, also im Sinne von "Aussehen", „erscheinen " usw.

Ich möchte die These aufstellen, daß es der Zusammenfall von „Widerspruch" und irgendwie legitimiertem Anspruch auf Sinnvollheit ist, die Prädizierung ,ist metaphorisch" (und ebenso, wie mir scheint, mit "Gleichnis, Allegorie, bildhafte Verwendung") rechtfertigt. Je nach dem Typ von Auflösung, den dieser Widerspruch erfährt, d. h. zu welchem Typ die Interpretationsprozedur gehört, wird die Etikette „Metapher", "Gleichnis", „Allegorie“ usw. zugeordnet. Wie diese Typen zu trennen sind, ist vorderhand noch eine Aufgabe, die ich vernachlässige bzw. der Literaturkritik überlasse.

Eine vorsichtige Einschränkung der These oben ist allerdings nötig: "Widerspruch" ist wahrscheinlich besser zu kennzeichnen als Nichtkompatibilität von semantischen Charakteristiken eines Teils des Satzes und dem Rest des Satzes - im Beispiel oben von alle Katzen sind schwarz mit in der Nacht sind alle Katzen schwarz. Black (1962: 28) spricht dabei von dem metaphorischen „Folsus" und dem „komplementären Rahmen" des Satzes, in dem dieser Folkus steht. Fokus kann auch - muß aber nicht, wie wir gesehen haben - ein einziges Wort sein. Blacks Beispiel dafür ist: 
(9) The chairman plowed through the discussion.

(was so viel heißt wie: „Der Vorsitzende leitete

die Diskussion nach eigener Vorstellung, ohne Rück-

sicht auf die Meinungen der anderen".)

Fokus ist hier allein das Lexem plowed.

Ganz ähnlich ist die Metapher in (4) zu verstehen.

(4) Der Mensch ist ein Wolf.

Wenn ich es recht sehe, dann setzt das Thema (der Mensch) eine bestimmte Selektionserwartung bezüglich der Merkmale des Prädikats, des Rhemas nämlich, das zu folgen hat. Darunter sind widersprüchliche Merkmale zu Mensch. Durch den Sieg der Erwartung der Sinnvollheit wird nach kompatiblen, aber nicht priorisierten (eventuell nicht nur nichtlexikalisch priorisierten, sondern nur enzyklopädischen) Merkmalen bei Mensch gesucht, die mit den lexikalischen (in der Terminologie von Black: common places im Wolfsystem) von Wolf verträglich sind - also: (Wild), (Gefräßig), (Blutrünstig).

Diese Suche ist erfolgreich: Unsere Erfahrung in dieser Welt erlaubt die Zuordnung von (Blutrünstig), (Wild), (Gefräßig) als definientes an das definiendum Mensch. Dieser Merkmalzuordnung sind Grenzen gesetzt. (Tier) wäre widersprüchlich, ebenso (Pflanze).

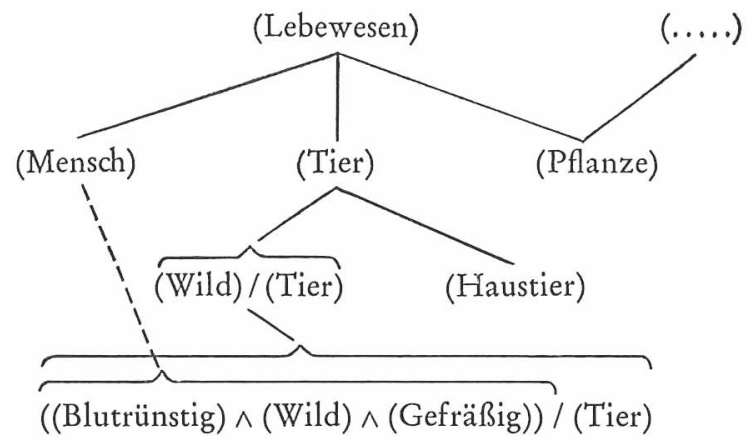

(Mit durchgezogenen Ästen gebe ich semantische implikative Beziehungen wieder, mit gestrichelten solche enzyklopädischen, die in einer bestimmten Welt aufgebaut werden können.)

Es ist anzunehmen, daß sich die Verwendung von solchen Metaphern wie Der Mensch ist ein Wolf nach folgenden Regeln vollzieht: Das Subjekt ist nicht nur das Satzthema, sondern es greift auch auf das auf; worauf in der Handlungssituation, die dem Sprechakt „metaphorischer 
Kommentar" vorausging, vorher Bezug genommen wurde. In diesem Sinne möchte ich die Anwendung der Identifikationsregel verstanden wissen als Wiederreferenz auf kontextuell (situativ oder geäußert) Bekanntes, während mittels der Prädikationsregel diesem Identifizierten eine neue Eigenschaft zugeordnet wird, die als fester Parameter für die durch die lexikalische Semantik festgelegte Verträglichkeit mit dem Argument gilt. So ist zu erklären, daß man eine sinnlos erscheinende Äußerung wie oben abweist mit [„Was du Unsinn sagst!] Der Mensch ist doch kein Wolf“, was soviel heißt wie: „Das Prädikat, das du dem Argument zugeteilt hast, habe ich überprüft, und es stimmt nicht; Mensch mußt du anders prädizieren".

Wie sieht das Erzeugen von priorisierten Merkmalen nach dem Parameter „ist Wolf“ aus? Offenbar durch die Priorisierung (Kurzschließung) von Implikationsketten. In diesem Sinne ist jede Metapher - im Vergleich zur wörtlichen Verwendung des Ausdrucks - eine weltschaffende Relation: „weltschaffend" weil sie andere Merkmalstrukturen setzt (enzyklopädische Merkmale miteinbezieht, andere Prioritäten in der Merkmalstruktur der wörtlichen Bedeutung setzt), „Relation" weil jeweils diese Umorganisation zu Metaphernbedeutung elizitiert wird durch die Beziehung zu einer wörtlichen Bedeutung des Metapherrahmens. Black (1962: 41) sagt zur Wolf-Mensch-Metapher: „... any human trait that can without undue strain be talked about in the "wolf-language" will be rendered prominent, and any that cannot will be pushed into the background. The wolf-metaphor supresses some details, emphasizes others - in short, organizes our view of man".4 Es ist klar, daß bei der Ubertragung von Merkmalen von Wolf auf Mensch der Mensch nicht alle Eigenschaften des Wolfes annimmt: so wird das Merkmal (Tier) ausgeschlossen, weil es mit (Mensch) unvereinbar ist. Insofern ist die Metaphernbildung (und erst recht die Metapherninterpretation) an Konventionen (Regelhaftigkeiten) geknüptt. Man kann keinen Sinn verbinden und daher auch keinen metaphorischen Sinn mit Das Blech ist ein Wolf, einfach weil die Enzyklopädien beider Begriffe keine kompatiblen dekompositionellen Einheiten haben - es sei denn das kleinste gemeinsame Implikat ist (Physisches Objekt); dies ist aber trivial und kann offenbar für Kompatibilitäten von enzyklopädischen Merkmalen nicht ausgenützt werden.

\footnotetext{
${ }^{4}$ Meine Sperrung; W. A.
} 
4. Bemerkungen zur Rolle der Begriffe "wörtliche Bedeutung" und „Akzeptabilität".

4.1. Zum Begriff „Priorisierung von Merkmalen“.

Was ich mit dem bisher nicht weiter erklärten Terminus „Priorität" von semantischen Merkmalen meine, soll an Hand eines Beispieles diskutiert werden. (Das Folgende ist technisch an einem Retrievalsystem orientiert. Es kann überschlagen werden bis zur Schlußfolgerung für das Metaphernproblem nach Beispiel ad 7). Der Nominalkomplex ist dabei einer Fachsprache des Strafrechts entnommen (Vgl. Abraham 1973). Diese Fachsprache wurde mit dem Ziel, ein information retrieval aufzustellen, nach Regeln, die in (1) illustriert werden, analysiert:

(1) Unzucht mit AвHängigen: (Geschlechtlich) / (Tun) $\wedge$ (Sittlichkeit) / / Fehlen) $\wedge$ (Selbständigkeit) / (Fehlen) / (Beziehung)

(Der Schrägstrich ist zu lesen als Funktor zwischen dem vorausgehenden Modifikanten und dem nachfolgenden Modifikat: also "geschlechtliches Tun“, „Fehlen der Sittlichkeit“ „Beziehung von Unselbständigkeit", wobei „Unselbständigkeit" weiter analysiert wird durch „Fehlen von Selbständigkeit"; es entsprechen also die ersten beiden Konjunkte der Bedeutung von Unzucht; mit Abbängigen wird wiedergegeben durch das dritte Konjunkt). In dem Beispiel wird folgendes sichtbar: Nach den allgemein verständlichen Regeln für den logischen Konjunktor " $\wedge$ " existiert absolute Symmetrie zwischen den Konjunkten. Die Prioritätsverhältnisse zwischen den Funktoren „/“ und „^" sind so, daß der Skopus des logischen Konjunktors genereller ist als der Skopus des /-Funktors. Offenbar kommen wir mit den normalen Konjunktoren der Logik deswegen nicht aus, weil im komponentenstrukturierten Aufbau von Wortbedeutungen dieses Formats Prioritäten gesetzt werden müssen. Würden nämlich aufgrund der Funktorenprioritäten in dem Beispiel oben Oberbegriffe gebildet, so würde (Fehlen) im zweiten Konjunkt als Oberbegriff zu dem Merkmalkomplex (Sittlichkeit)/(Fehlen) übrig bleiben. Dies ist jedenfalls eine sehr unbefriedigende Charakterisierung des Themas, um welches es bei der Implikation (Sittlichkeit)/(Fehlen) $\supset$ (Fehlen) geht. Denn in der Darstellung der Privativität von ,Unselbständigkeit' ist nichts das privative Element hier (Fehlen), sondern (Selbständigkeit) der „Träger der Bedeutung" („Kern“, „Thema“; in einem Retrieval-system der „Deskriptor", unter dem man nach dem Privativum, dem Positivum sowie Modifikaten davon sucht). 
Aufgrund allgemeiner Beobachtungen innerhalb unseres Systems haben wir nun solche Merkmale wie (Fehlen) zu einer sogenannten Klasse der „Funktionswörter" zusammengefaßt, die bei Ableitung von Oberbegriffen jeweils zuerst getilgt werden. Aufgrund dieses ersten Prinzips heißt also das erste Ableitungsprodukt der Oberbegriffbildung zu (1):

(2) $($ Geschlechtlich $) /($ Tun $) \wedge($ Sittlichkeit $) \wedge($ Selbständigkeit $) /($ Beziehung)

Und aufgrund des letzten Schrittes:

(3) (Tun) $\wedge$ (Beziehung) $\wedge$ (Sittlichkeit)

Aber auch dies ist unbefriedigend, da (Tun) und (Beziehung) die Definienda Unzucht bzw. AвHÄNGIGKeIt nur sehr schwach thematisieren. Wir wollen daher zwei weitere Prioritäten einführen: eine die durch die Thematisierung auf der Komponentenebene gegeben ist sowie eine die durch Thematisierung auf der Merlsmalsebene gegeben ist.

Illustration zur Thematisierung auf der Komponentenebene: (steigende Priorität wird durch steigende Zahlen vor den Komponenten gekennzeichnet):

(4) Unzucht mit Abhängigen: 2(Geschlechtlich/Tun) $\wedge$ 2(Sittlichkeit/Fehlen) $\wedge 1$ (Selbständigkeit/Fehlen/Beziehung)

Illustration zur Thematisierung auf Merkmalsebene:

(5) Unzucht mit AвнÄNGigen: 1Geschlechtlich/Tun $\wedge$ 1Sittlichkeit/Fehlen $\wedge$ 2Selbständigkeit/Fehlen/Beziehung

Beim Prozeß der Oberbegriffbildung müssen also die Prioritäten in folgender Rangordnung berücksichtigt werden:

1) Merkmalspriorität

2) Komponentenpriorität

3) Formale Priorität

Die Merkmalspriorität berücksichtigt dabei, daß Elemente der Klasse der Funktionswörter jeweils Nullpriorität besitzen, d. h. nachrangig gegenüber allen anderen Elementen sind.

Illustration eines Prozesses zur Oberbegriffsbildung unter Berücksichtigung dieser drei Prioritäten:

(6) Unzucht mit AвHÄNgigen

(6a) $2(1$ Geschlechtlich/Tun $) \wedge 2(1$ Sittlichkeit/Fehlen $) \wedge$

$\wedge 1$ (2Selbständigkeit/Fehlen/1Beziehung).

(6b) $2(1$ Geschlechtlich/Tun $) \wedge 2(1$ Sittlichkeit/Fehlen $) \wedge$

$\wedge$ 2Selbständigkeit/1Beziehung

(6c) $2(1 \mathrm{Geschlechtlich/Tun)} \wedge 2(1$ Sittlichkeit/Fehlen $) \wedge$ $\wedge$ Selbständigkeit 
(6d) $2(1$ Geschlechtlich/Tun) $\wedge 2($ Sittlichkeit/Fehlen)

(6e) 1 Geschlechtlich/Tun $\wedge 1$ Sittlichkeit/Fehlen

(6f) Geschlechtlich $\wedge$ Sittlichkeit

Das heißt, daß die Ordnung der Proposition (Prädikationen) in einer Deskriptionsliste wie dem Thesaurus - der Grundlage für ein Retrievalsystem - nicht arbiträr ist. Die Darstellung mithilfe von Ordnungsziffern ist natürlich nur eine von mehreren möglichen. So könnte z. B. auch eine Rangordnung durch die links-rechts-Abfolge zum Ausdruck gebracht werden. Es sollte in diesem Beispiel aus der Dokumentationswissenschaft gezeigt werden, daß es plausibel ist, zwischen logischen Konjunkten Prioritäten anzunehmen. Dies geschah hier mit dem Ziel, die notwendigen kleinsten Schritte bei der Oberbegriffsbildung zu beschreiben. Bei der Metapherninterpretation geht es offenbar darum, Merkmale, die die Normalbedeutung eines Lexems nicht haupträngig hergeholt werden müssen, zur Kompatibilisierung der Lexeme heranzuziehen. (Ich betrachte dies als weiterführende Diskussion der Unterscheidung von "nuklearen" und "peripheren" semantischen Merkmalen, wie sie von Bierwisch und Kiefer (1969: 72) getroffen wird. Den Gedanken über Ordnungen von semantischen Merkmalen (dort Ordnungen von Hypothesen über die Anwendbarkeit eines lexikalischen Elements) treffen auch Cohen/Margalit 1970, bes. $476 \mathrm{ff}$.) Vgl. die Diskussion der Literatur am Ende von 4.2.) Die Norm über die Bedeutung eines Wortes (eines Lexems) ist eine geordnete Menge von Hypothesen über die richtige Verwendung dieses Wortes, die sich über längere Zeit, in der sie der Verwendung in einer Sprachgemeinschaft ausgesetzt sind, nicht widerlegen lassen.

Die Hypothesen sind konkret aufzufassen als Wahl zwischen Paaren von Prädikaten, deren Elemente einander komplementär ausschließen von Prädikaten, die die Eigenschaft, die durch das Lexem bezeichneten Ding zugesprochen wird, bestätigen. Solche Prädikatenpaare sind wie gesagt kontradiktorisch, also entweder Antonyme wie schnell/langsam oder durch Negierung zu fassende Gegensätze wie [ \pm lebendig].

Daraus geht hervor - was durchaus den Erkenntnissen (schon) der (traditionellen) Sprachwissenschaft entspricht -, daß „wörtliche Bedeutung" (= Bedeutungsnorm) nicht fest ist, sondern offenbar vom einzelnen Sprecher einer Sprache in seinem eigenen sprachgenetischen Prozeß induktiv erschlossen und (bewußt oder unbewußt) ständig auf ihre Haltbarkeit hin überprüft wird. Katachrese, diachronischer Bedeutungs- 
wandel sind Resultate des Verlustes des Normstatus von Wörtern oder lexikalisierten Phrasen.

Solche eine Norm gilt offenbar nur für den Namen oder die Relationsbeschreibung der Klasse von Dingen, die eine möglichst große und relevante Menge von Sprechern in einer Sprachgruppe mit großer Frequenz gebrauchen. Neben dieser durch Verwendungsfrequenz fixierten Norm steht die durch Definition fixierte Norm, so wie sie in reinen Kunstsprachen und z. T. (je nach Anteil an Umgangssprache stärker oder schwächer) in Fachsprachen vorliegt.

Was ist nun die philosophische Grundlage der „Erklärung“" oben: „Bedentungsmerkmale durch enzyklopädische Merkmale ersetzen?" Auch hier liefert Black meines Erachtens einen Zugang zur Antwort (Black 40). An seine Begründung des Begriffs „system of associated common places" lassen sich folgende Überlegungen anschließen: Wörtliche Verwendungen eines Wortes (die Standardbedeutung, die Lexikonbedeutung) ist eine in der ständig vollzogenen und daher ständig überprüfbaren sprachlichen Kommunikation sich konstituierende Menge von Meinungen über das (Haltungen zum) Designatum des Lexems. Solche Meinungsmengen müssen gruppentypisch und in solchen Gruppen in einem naiven statistischen Sinne signifikant sein. Sie können dabei durchaus genïgend oder widersprüchlich sein, gemessen an den „Meinungen" anderer Analysen: etwa einer Fachsprache, der sich bestimmte Experten bedienen. Diese gruppentypisch relevante und signifikante Menge von Meinungen ist innerhalb der betreffenden Gruppe jene Menge, die mit den geringsten Einschränkungen (hinsichtlich Kontext, Situation) evoziert werden kann. Sie entspricht jener Menge, die jeder Vertreter beim anderen voraussetzen kann (sie entspricht der Erwartungsnorm: zum Terminus vgl. Abraham-Braunmüller 1971). Man sieht, daß es so geschehen sinnvoll ist, von Bedeutungen von isolierten Lexemen zu sprechen und diese gegenüberzustellen Bedeutungen, die erst durch bestimmte Kontexte und Situationen evoziert werden. Die Suche nach einer Interpretation einer inkompatiblen Lexemkookkurrenz ist die Kuche nach einem Kontext (nach einer zu der vorgegebenen alternativen Welt), der diese Lexemkookkurrenz kompatibel macht.

$\mathrm{Zu}$ den Prioritätsverschiebungen in unseren Beispielen:

Jedes Merkmal zwischen ein Klammerpaar. "^“ ist der logische Konjunktor, „V" steht für "und/oder". Die Redundanz von Merkmalen wird nicht vermieden, da das Merkmal hier nur mit dem Ziel ver- 
wendet wird, die Assoziationen des Wortgebrauchs inbezug auf eine bestimmte Welt (die der Umgangssprache, so wie ich sie gebrauche) zu illustrieren; in dieser Funktion ist auch der Doppelpunkt zwischen Definiendum und den Verknüpfungen von definierenden Merkmalen zu verstehen.)

ad (7) [wobei dahingestellt bleibt, ob dieses Beispiel als Metapher zählt]:

KATZE: $(($ Säugetier $) \wedge($ Haustier $) \wedge($ Felltier $) \wedge$ (Mäusefresser $) \wedge$ $\wedge((($ Weiß $) \vee($ Braun $) \vee($ Gefleckt $) \vee \ldots \vee($ Schwarz $)) /$ $($ Farbe $) \wedge \ldots)$

$\Rightarrow$

$(($ Schwarz $) \wedge \ldots)$ im Kontext $(($ Schwarz $) /($ Nacht $)$

[Das Merkmal (Schwarz) gehört zu den "common place associations" von NACHT; regeltechnisch gesprochen heißt dies, daß das Merkmal über eine Redundanzregel verfügbar wird: (Nacht) つ (Schwarz)/ (Nacht))]

$\operatorname{ad}(4):$ Mensch: $($ Säugetier $) \wedge($ Mensch $) \wedge($ Zweibeinig $) \wedge($ Sprache $) \wedge$ $\wedge($ Vernunft $) \wedge($ Verstand $) \wedge \ldots$

$\Rightarrow \quad($ Wild $) \wedge($ Aggressiv $) \wedge($ Gefräßig $) \wedge(($ Blutrünstig) $\wedge \ldots)$ im Kontext WolF

wobei WoLf: (Wild $) \wedge($ Aggressiv $) \wedge($ Gefräßig $) \wedge$ (Blutrünstig $) \wedge$ $\wedge($ Säugetier $) \wedge($ Hund $) \wedge($ Vierbeinig $) \wedge \ldots$

[Die Inkompatibilität der Relation (Mensch) $D$ (Hund) sowie (Hund) $D$ $D$ (Mensch) wird duch die Prioritätsverschiebung aufgehoben]. $\operatorname{ad}(1)$ : Sich DURChFressen: $(($ Mensch $) \vee($ Tier $)) \wedge($ Kauen $) \wedge$

$\wedge($ Verdauen $) \wedge(($ Eßbar $) /($ Nahrung $)) \wedge($ Menge von Nahrung) $\wedge(($ Sehr viel $) /($ Nahrung $)) \wedge$ $\wedge(($ Mühevoll $) /($ Arbeit $)) \wedge \ldots$

$\Rightarrow \quad(($ Mühevoll $) /($ Arbeit $))$ im Kontext BERG voN KorRespondenz wobei Berg von KorresponDENZ: $($ Brief $) \wedge(($ Viel $) /($ Arbeit $)) \wedge(($ Schreiben $) \vee$ $\vee($ Diktieren $)) \wedge \ldots$

wobei ferner ein Bedeutungspostulat gelten muß wie $(($ Viel $) /($ Arbeit $)) \supset$ (Mühevoll)/(Arbeit))

Dies gilt übrigens nicht umgekehrt, jedenfalls nicht in der Welt, die hier zugrundeliegt: (Mühevoll)/(Arbeit)) /(Viel)/(Arbeit)).

Die Kompatibilitäten in den Beispielen (2), (3) und (5) sind ähnlich abzuleiten.

Komplexer ist (6). Hier handelt es sich offenbar um wenigstens zwei 
Inkompatibilitäten: einmal (Hell) Licht: (Dunkel) Schatten: und zum anderen um die ausgedrückte Identitätsrelation LICHT = SCHATTEN, dem das folgende Bedeutungspostulat gegenübersteht:

\section{Schatten $\supset$ Licht}

wobei hier Licht als Lichtouele verstanden wird und das Implikationszeichen zu lesen ist als „setzt voraus“. Beidemale ist die Nichtvereinbarkeit durch die Bezeichnung auf Gотт aufzulösen: einmal durch das priorisierte Merkmal ((Absolut)/(Klarheit)) für GotT wobei das weitere Voraussetzungspostulat (Klarheit) $\supset$ (Sichtbarkeit) $\supset$ (Helligkeit) angestrengt werden könnte; das andere Mal muß für GoT'T offenbar eine Charakteristik wie (Quelle allen Lichts) priorisiert werden, erschließbar aus dem Merkmal (Weltschöpfer). Meiner Interpretation nach ist noch eine dritte Unvereinbarkeit aufzulösen: SchATTEN hat ein Merkmal (Kontour eines Objekts, Licht hingegen nicht; dieses wird erst aktualisiert durch die Aktualisierung des Prädikats "Gott ist die Quelle allen Lichts" oben. (Es scheint damit eine Ordnung zwischen den Merkmalaktualisierungen in diesem speziellen Fall vorzuliegen; ich bin aber nicht in der Lage zu sagen, ob diese Beobachtung irgendeine generellere Bedeutung hat).

\section{2. Überlegungen zur Akzeptabilität und Grammatizität}

Metaphern sind Sätze oder syntaktische Strukturen, die (mehr oder weniger) akzeptabel, aber jedenfalls sinnvoll sind. Und zwar hängt die Interpretierbarkeit offenbar von dem Grad der Akzeptabilität ab. Man kann Akzeptabilität als Sonderfall der Grammatizität betrachten und zwar insofern, als grammatische Sätze oder komplexe Konstituenten (wie das Bein des Tisches, das Bein des Menschen) zu einem bestimmten Zeitpunkt des sprachlichen Leistungsvermögens aus allen zu diesem Zeitpunkt gespeicherten Sätzen oder komplexen Konstituenten bestehen plus den aufgrund der Grammatik ableitbaren Sätzen oder Konstituenten, wenn alle möglichen generativen Regeln rekursiv über der Menge der gespeicherten Sätze wirksam werden (Kintsch 1972: 278 ff.) Die semantische Wohlgeformtheit dieser Sätze wird durch eine zu diesem Zeitpunkt geschlossenen Menge von Bedeutungspostulaten kontrolliert. Für jedes einmalige Durchlaufen aller Erzeugungsregeln unter Wahrung einer bestimmten Menge von Bedeutungspostulaten erhält man eine Menge von Sätzen, die semantisch akzeptabel sind. D. h. wenn $\left\{\mathrm{P}^{\mathrm{t}}\right\}$ die Menge aller zum Zeitpunkt durch die Grammatik re- 
kursiv erzeugten Sätze ist, dann ist ein beliebiger Satz Q seman tisch akzeptabel, wenn $\mathrm{Q} \varepsilon\left\{\mathrm{P}^{\mathrm{t}}\right\}$.

(16) $\quad \forall Q\left(Q \varepsilon\left\{\mathrm{P}^{\mathrm{t}}\right\} \Leftrightarrow\right.$ Aкzept $\left.(\mathrm{Q})\right)$

Wenn diese Menge $\left\{\mathrm{P}^{\mathrm{t}}\right\}$ von Untermengen mit steigendem Umfang $\left\{\mathrm{Pt}_{\mathrm{i}}\right\}$ (d. h. von Satzmengen mit jeweils geänderten Mengen von Bedeutungspostulaten) durchlaufen wird, dann können wir den Index „i“ als Akzeptabilitätsmaß angeben: Je größer i, desto weniger akzeptabel wird $\mathrm{P}_{\mathrm{i}}$. ( $\left\{\mathrm{P}_{\mathrm{o}}{ }_{\mathrm{o}}\right\}$ ist die Menge der zum Zeitpunkt $\mathrm{t}$ im Gedächtnis gespeicherten Sätze, wobei $\phi \leqq \mathrm{g} \leqq \mathrm{i}$.)

Das alles ist die (sehr grob formulierte) These, daß die direkt gespeicherten Sätze $\left\{\mathrm{P}_{\mathrm{o}}\right\}$ und die daraus grammatisch ableitbaren aufgrund eines zum Zeitpunkt $t$ bestehenden Lexikonzustands gebildet werden. Ein solcher Lexikonzustand würde z. B. folgende Bedeutungspostulate umfassen:

$$
\left.\begin{array}{l}
\text { (Trommeln) } \supset \text { (Dumpf)/(Ton) } \\
(\text { Trommel }) /(\text { Schlagen }) \equiv(\text { Trommeln) }
\end{array}\right\} \text { Lexikonregeln }
$$

Um nun die metaphorische Verwendung von (3) in 3.1. interpretierbar zu machen, muß eine neue Regel hinzugefügt werden, die offenbar aus dem enzyklopädischen Wissen, der mehr oder weniger individuellen Erfahrung aus unserer Welt stammt.

(3) Der Regen trommelt auf das Dach.

(18) $((($ Hart $) \wedge($ Schwer $) /($ Fallen $) /($ Regen $)) \rightarrow($ Dach $)) \supset$ (Dumpf)/(Ton)

Wenn diese Regel oft genug produziert wird und antizipiert wird, geht sie in den Bestand der Lexikonregeln zu irgendeinem Zeitpunkt über. Das ist der Zeitpunkt, wo eine solche Metaphernverwendung auch in ein Wörterbuch hineingehört. Alle anderen Metapherninterpretationen müssen jeweils neu über die Enzyklopädie erbracht werden (vgl. Abschnitt 4.1.).

Eine weitere Illustration. Offenbar ist (19) akzeptabler als (20)

(19) Fressen (Katze, Maus) $\in\left\{\mathrm{P}_{0}\right\}$

(20) Fressen (Maus, Katze) $\in\left\{\mathrm{P}_{\mathrm{k}}\right\}$ für $\mathrm{k}>\phi$

Offenbar sind folgende oder ähnliche Bedeutungspostulate dafür verantwortlich:

(21) für $P_{0}$ Maus: (+ Futter für Katze) $\supset(+$ Eßbar $)$

für $P_{k}$ KATZE: ( - Futter für Maus) $\wedge(+$ Futter für größere

Tiere als die Maus). $\supset(+$ Eßbar $)$

(22) für $P_{k+n}$ Wolle: $(-$ Eßbar $) \wedge(+$ Verschlingbar $) . \supset(+$ Verschlingbar) 
(23) für $P_{k+r}$ Haus:

$(-$ Eßbar $) \wedge(- \text { Verschlingbar })_{\text {[für normale Katzen und Mäuse] }} \wedge$

$\wedge(+ \text { Verschlingbar })_{\text {[für Riesenmäuse] }}$

(22) und (23) erklären die sinkende Akzeptabilität der folgenden Sätze (indiziert wird sinkende Akzeptabilität dadurch, daß man Ausdrücke wie $\left(22^{\prime}\right)$ und (23') kommentiert mit „unter Umständen" und eventuell diese Umstände angibt: für $\left(22^{\prime}\right)$ : „Sie war halbverhungert"; für $\left(23^{\prime}\right):, \ldots$ in einem Micky-Maus-Abenteuer").

(22') Die Katze frißt die Wolle.

(23') Die Maus verschlingt das Haus.

Die jeweils zweiten Konjunktionsglieder in den Merkmalverkniipfungen von Katze, Wolle und das 3. Konjunktionsglied in Haus sind aus der Enzyklopädie des Lexikonzustands $t_{1}$ zugeordnet, um die Sätze $\left(22^{\prime}\right)$ und $\left(23^{\prime}\right)$ (verschiedengradig) akzeptabel zu machen.

Ich fasse die Annahmen zusammen - und gehe bei dieser Gelegenheit auf einige neuere linguistische Arbeiten zur Metapher ein:

4.2.1. Es gibt kein stabiles Lexikon in dem Sinn, daß sich Akzeptabilitäten nicht ändern könnten. Hier spielt der Rückkoppelungsprozeß vor allem durch Massenmedien eine sehr starke Rolle. Daraus geht auch hervor, daß das Lexikon abhängig ist vom enzyklopädischen Wissen. (Diese Annahmen trifft in ähnlicher Weise Bickerton 1969).

4.2.2. In diesem instabilen Lexikon gibt es jeweils nur Konsistenz zwischen partiellen Bedeutungsbereichen: solchen die gerade aktuell abrufbar sind. Diese Konsistenz ist möglicherweise in extremen Fällen beschränkt auf einzelne binäre Oppositionen.

4.2.3. Im besonderen zeichnet sich das instabile Lexikon aus durch Varianz der Implikationsreihen: die Anzahl der Implikationselemente zwischen den maximalen und minimalen Gliedern kann wechseln $(=$ in Aktualität variabel sein).

4.2.4. Metapher ist ein Kontextphänomen - ebenso wie Abweichung (= Selektionsregelverletzung) -, während Normalbedeutung auch ein Problem des Einzellexems ist (der Kernbedeutung, des "Archilexems").

D. h. metaphorische Bedeutung - im Gegensatz zur syntaktischen Abweichung - kann nur durch Kontextbezug (innerhalb der Satzgrenzen wie auch darüber hinaus) elizitiert werden. Wesentlich für den Abruf eines Versuchs, eine Inkompatibilität metaphorisch zu interpretieren, scheint weiter die Erwartung zu sein, die man bezüglich der Sinnvollkeit einer Äußerung eines Kommunikationspartners hat.

4.2.5. Die einzig sinnvolle kategorische Unterscheidung scheint mir die 
zu sein zwischen abweichenden und nichtabweichenden Sätzen einerseits und sinnvollen und sinnlosen Sätzen andererseits. Nur wo "abweichend" und „sinnvoll" verknüpfbar werden, liegt eine übertragene Bedeutung (nicht notwendigerweise nur Metapher) vor.

4.2.6. Wenn in dieser Verknüpfung das Merkmal "abweichend“ ( = „nicht hochgradig akzeptabel“) durch häufigen Gebrauch in der Akzeptabilität steigt - wenn also die Verknüpfung „akzeptabel-sinnvoll“ entsteht -, dann wird daraus ein Metaphernklischee. D. h. noch nicht, daß die WIE-Relation herstellbar sein muß. Es entsteht einfach eine neue Bedeutung für ein Lexem in einem bestimmten (im Lexikon verzeichneten) Kontext.

Ich möchte einen grundsätzlichen Unterschied machen zwischen „Metaphernklischee" und Metapherninnovation. Eigentlich sollte man „Metaphernklischees" nur dann als Bezeichnung wählen, wenn die WIERelation herstellbar ist, anderenfalls handelt es sich um eine neue Bedeutung eines Lexems in einem bestimmten Kontext.

Bei der Beobachtung oben über die Merkmale „abweichend" und „sinnvoll" sollte es in 4.2.5. nicht um eine symmetrische logische Verknüpfung der Merkmale handeln: Es ist vielmehr so, daß die Abweichung eine Funktion der Interpretierbarkeit (Sinnvollheit) wird, um Metapher zu werden. Das heißt durch „Kontextsog" und „Erwartung" (jeweils höchst pragmatische Phänomene) wird die Funktion auf ihr(e) Resultat(e) hin überprüft. Mit dem Erkennen der Interpretierbarkeit ändern sich nicht die Kompetenzregeln, sondern es wird nur nach (linguistisch-enzyklopädisch jeweils zu spezifizierenden) Regularitäten Sinnvollheit zugeordnet. Dies ist ein Prozeß der systematischen Sprachverwendung (im Einklang mit Matthews 1971). Daß dies für den Metaphernschöpfer systematisch ablaufen muß, ist klar - es gibt ja auch sinnlose Sätze.

4.2.7. Es scheint mir für den Aufbau des Lexikons unwesentlich zu sein anzunehmen, daß

4.2.7.1. es Primitiven gibt, solange es nicht spezifisch Argumente gibt, die Annahme von Primitiven in der systematischen Sprachverwendung rechtfertigen. Die Lexeme können sich durchaus rekursiv und gegenseitig definieren.

4.2.7.2. Es muß in jedem Lexikonzustand spezifiziert werden, wie die Oberbegriffs-Unterbegriffsrelationen aussehen. Ohne eine Theoretisierung dieser Konzepte kommt eine Theorie des Lexikons nicht aus, will 
sie nicht der Intuition zurïckbleiben bzw. ihr widersprechen (dies ist eine deutliche Kritik an Matthews 1971).

4.2.7.3. Die Merkmale sind - und im Einklang mit dem Konzept des wenigstens teilweise instabil organisierten Lexikons - Illustrationen für die häufigste(n) Verwendung(en) des Lexems. Die spezifischen Merkmale und ihre Struktur sind demnach Generalisierungen über den Gebrauch des Lexems in Kontexten, die zu den jeweiligen Generalisierungen passen. Wir können auch sagen, daß es Prädikate sind, mit Hilfe derer die Verwendung des Lexems bezüglich der entsprechenden Kontexte verifiziert werden kann. Der theoretische Begriff „Merkmal“ ist dann eine Prädikatskategorie, mit Hilfe derer Verwendungen von Lexemen bezüglich ihrer entsprechenden Kontexte verifizierbar sind (vgl. Abraham 1972: 6 [im Einklang mit Bartsch 1971]). Das räumt - meine ich - auch die eher komisch anmutenden Bemerkungen über „Kompetenzen von Sprechern im Merkmalesischen“ weg (Coseriu, Heringer).

4.2.8. Zu sagen, daß the rock is becoming brittle with age keine Metapher ist, weil diese Abweichung kein Selektionsregelverstoß ist (Matthews 1971: 423), ist - gelinde gesagt - bequem. Ich möchte eine andere Erklärung suchen. Sie ist zu erreichen, indem man nicht apodiktisch fordert, daß nur Selektionsabweichungen Metaphern charakterisieren, sondern indem man bei Abweichung den generellen Verstoß gegen lexikalische Kompatibilität mitberücksichtigt. Ich betrachte deswegen die Angaben zu den notwendigen und hinreichenden Bedingungen für eine Metapher bei Matthews als falsch (Matthews 424). Ihre Unhaltbarkeit wird deutlich bei ist-Prädikationen wie Der Mensch ist ein Wolf, Der Ton ist kalt usw.; dafür nämlich sind Selektionsregelverstöße nicht definierbar, wohl aber Abweichung von der allgemeineren Kompatibilitätsregel. Die Bedingungen sind nicht hin reichend, weil Erwartung und Kontext (alternative Welten) die Art der Neuorganisation der semantischen Merkmale bestimmen bzw. überhaupt erst über Kompatibilität oder Nichtvereinbarkeit der lexikalischen Elemente entscheiden. 4.2.9. "The metaphorical meanings of a word or phrase in a natural language are all contained as it were, within its literal meaning or meanings. They are reached by removing any restrictions in relation to certain variables from the appropriate section or sections of its semantical hypothesis." (Cohen/Margalit 1970: 482). Das heißt, daß man bei der Konstitution der (Normal-)Bedeutung eines Wortes alle jene Merkmale („Variablenpaare“ bei Cohen/Margalit) mit einfließen läßt, 
die einmal - bei gegebener Assoziation mit Teilen aus alternativen Welten, kurz aus der Enzyklopädie - bei einer Metapherninterpretation relevant werden können. Das ist natürlich nicht möglich, denn die apriori nicht lexilkalisierte Interpretabilität bzw. Kontextkompatibilität eines Wortes ist das Wesen der Metapher; andernfalls wäre es einfach eine andere Bedeutung, als Homonymie kodifiziert. Cohen/ Margalit selbst sprechen von einer Ordnung der Bedeutungskonstituenten für jedes Lexem, die bei metaphorischer Interpretation des Lexems umstürzt: "Note that in all such cases the variable or variables that have their restrictions removed may be expected to be fairly near the beginning of the ordered sequence of relevant variables. Any such variable must be a fairly important one." Vgl. auch Cohen 1971, 684: „... an order of relative importance has in any case to be supposed for the set of distinctive features that characterize a particular meaning. So the less important features will normally be the ones that are exposed to cancellation or deletion in literal usage". Wenn in dieser Ordnung (Priorisierungsverhältnis in meiner Terminologie) der Ưbergang zwischen lexikalischen und enzyklopädischen Bedeutungskomponenten zum Ausdruck kommt (vgl. Bierwisch/Kiefer 1969, 72, wo die Begriffe "semantic core" und „semantic periphery of a lexical entry" entwickelt werden und der Scheidung zwischen sprachlichem (linguistic) und enzyklopädischem Wissen gleichgesetzt wird. Es fehlen dort allerdings explizite Hinweise darauf, daß Ordnung unter den Merkmalen im Sinne von gradueller Verifikationsmöglichkeit für die Anwendung des Lexems vorliegen muß, um Prozesse der Bedeutungsübertragung im speziellen, den Wechsel zwischen alternativen Welten (Zustandsbeschreibungen nach Carnap) im allgemeinen erklären zu können, dann kann die metaphorische Bedeutung nicht in der lexikalischen enthalten sein - nach den konventionellen Anwendungsbedingungen des Wortes enthalten (contain).

Cohen/Margalit (1970: 485) kommen zu dem Schluß, daß es in natürlichen Sprachen überhaupt keine semantisch abweichenden Sätze gibt. Was sie damit sagen wollen, ist offenbar, daß zu jeder semantischen Anomalie ein Kontext gefunden werden kann, der eine metaphorische Interpretation gestattet. Das geht auch aus seiner prinzipiellen Klassifizierung aller syntaktisch wohlgeformten Sätze in nur metaphorisch zu interpretierende und sowohl metaphorisch wie auch nicht-metaphorisch (wörtlich) zu interpretierende hervor. Gegen diese Annahme spricht: Wenn die Interpetier- 
barkeit einer Wortverwendung als Metapher davon abhängt, daß ein bestimmter Kontext (eine alternative Welt) gefunden wird, dann kann jedenfalls nicht ausgeschlossen werden, daß bestimmte Sprachverwender die nötigen alternativen Welten (Enzyklopädien) nicht kennen, d.h. die Wortverwendung nicht sinnvoll interpetieren können. Es ist also jedenfalls pragmatisch nötig - wollen wir nicht die Wirklichkeit um eines rein deduktiven Vorgehens willen opfern -, auch syntaktisch wohlgeformte, aber sinnlose (semantisch abweichende) Sätze zu klassifizieren sowie der Mühe Rechnung zu tragen, die es manchmal kostet, hinter den Sinn einer abweichenden Struktur zu kommen.

\subsection{Alternative Welten, Wahrheit und metaphorische Prozesse}

Um die hier angeschlagene Thematik motivieren zu können, muß ich vorausschicken, was die entscheidende Charakteristik metaphorischer Prozesse ist: Bei metaphorischen Prozessen (bzw. allgemeiner bei Prozessen der Bedeutungsübertragung) werden Bedeutungspostulate, die für einen bestimmten Lexikonzustand gültig sind, aufgegeben bzw. durch zu diesem ursprünglichen Lexikonzustand widersprüchliche ersetzt.

Lexikonzustände unter Aufgabe bestimmter Postulate bzw. mit jeweils neuen Postulaten, die bestimmte Relationen zwischen den einzelnen Lexemen herstellen, sind vergleichbar mit den "Zustandsbeschreibungen (state-descriptions)" bei Carnap, den "möglichen Welten" bei Leibnitz und den "möglichen Zuständen (possible states of affairs)" bei Wittgenstein (Tractatus logico-philosophicus). Die Beschreibung eines solchen Zustandes wird geliefert mit Hilfe der Sprache der Symbollogik, etwa der, wie sie Carnap entwickelt hat (Meaning and Necessity). Das heißt, alle Relationen zwischen den einer bestimmten Zustandsbeschreibung angehörigen Lexembedeutungen wird mithilfe der in einer solchen Symbolsprache üblichen Konnektoren und Operatoren dargestellt. Da diese Bedeutungsrelationen jeweils nur für eine bestimmte Zustandsbeschreibung (eine bestimmte alternative Welt) gültig sind, muß vorerst festgelegt werden, welches die Bedingungen dafür sind, daß eine bestimmte einfache Aussage (ein atomarer Satz, wie Carnap sagt) zu einer solchen Zustandsbeschreibung gehört. Diese einfachen Aussagen (atomaren Sätze) setzen sich zusammen aus $n$-stelligen Prädikaten, gefolgt von jeweils $n$ Individuenkonstanten; es sind nur Behauptungssätze, symbolisiert etwa so: $\mathrm{P}(\mathrm{a}, \mathrm{b})$, wenn $\mathrm{P}$ ein zweistelliges Prädikat ist. Mithilfe dieser einfachen Aussagen soll der tatsächliche Zustand einer 
ganzen Welt beschrieben werden. Alle einfachen Aussagen (atomaren Sätze), die die Gesamtheit dieser Welt beschreiben, werden in einer $\mathrm{Zu}-$ standsbeschreibung zusammengefaßt. Sie sind also in dieser Welt wahr. Wie behandeln wir nach so einem Konzept semantische Anomalien oder Bedeutungsübertragungen (Metaphern)? Betrachten wir dazu die folgenden Beispiele:

(1) Der Junggeselle ist unverbeiratet

Danach - vorausgesetzt (1) ist ein analytischer Satz - lassen sich die folgenden Bedeutungspostulate aufstellen:

(2) Junggeselle $\supset$ unverheiratet

(2') Junggeselle $\supset$ verheiratet

Für jede Anomalie bzw. Bedeutungsübertragung, die solchen Bedeutungspostulaten widerspricht (wie $\left(2^{\prime}\right)$ ), sind prinzipiell zwei Erklärungsmöglichkeiten offen:

(a) Ein Satz, z. B. (1) ist in der Welt von (1) $\left(W_{1}\right)$ analytisch und (notwendig) wahr, in der Welt von (3) $\left(\mathrm{W}_{3}\right)$ hingegen nicht (also entweder synthetisch oder aber wohl analytisch, jedoch falsch).

(b) Eine semantische Anomalie in $W_{1}-$ die in $W_{1}$ auf einen atomaren Satz mit dem Prädikat „falsch" zurückgeführt werden kann - erhält eine semantische Interpretation ( = wird $\mathrm{W}_{1}$-wahr), wenn Bedeutungspostulate wie (2) bzw. (2') nicht mehr gelten.

Der Verfall solcher Bedeutungspostulate in solchen einzelnen Fällen von semantisch notwendiger Inkompatibilität (wie etwa verbeiratet und Junggeselle) ist aber nicht ein Wechsel von $\mathrm{W}_{1} \mathrm{zu}_{3}$ (mit dem Bedeutungspostulat $\left(2^{\prime}\right)$ ), sondern setzt eine Änderung der Intension eines der inkompatiblen Wörter in einer Weise voraus, die ich unter dem Begriff „Priorisierung von Bedeutungskomponenten" erfasse.

Überlegen wir die Thesen (a) und (b) an Beispiel (3).

(3) Er ist ein verbeirateter Junggeselle

[Dafür kann ich mir folgenden Sinn zurechtlegen: „Er ist zwar qua lege verheiratet, und man würde daraus erwarten, daß er bestimmte Familienpflichten übernimmt, aber er gebärdet sich (weiter) wie ein ungebundener Junggeselle."]

Unter These (a) ist die Relation

(2') Junggeselle $\supset$ verheiratet

entweder nicht determinierbar (Fall „C"); d. h. wir dürfen nicht annehmen, daß die Relation gilt, da wir nicht wissen, ob (3) eine analytische oder eine synthetische Aussage ist. Oder aber $\left(2^{\prime}\right)$ ist in der Welt determiniert, in der (3) eine analytische Aussage ist (Fall „D"): dann 
ist es widersprüchlich gegenüber (2), d. h. es wäre in der Welt, in der (1) gültig ist, nicht möglich. Die beiden Welten sind nicht kompatibel miteinander; ihre Semantiken sind zumindest im Bereich der Wirklichkeitsbeschreibung von (1) und (3) in diesen alternativen Welten sowie in allen daran anzuschließenden Implikationen widersprüchlich. Aus D ist zu folgern, daß (3) keine Bedeutungsübertragung von (1), d. h. kein sinnvoller Satz in bezug auf die Welt von (1) sein kann. Es folgt weiter daraus, daß entweder (im Fall C) die Carnapsche L-Determiniertheit nicht zutrifft (Durchschnitt aller Determiniertheiten in anderen Zustandsbeschreibungen) oder eben daß (im Fall D) für (1) und (3) nicht L-Wahrheit gilt (Wahrheit in allen Zustandsbeschreibungen).

(3) ist nun deutlich auch metaphorisch interpretierbar. Und ich möchte am Beispiel der Metapher (und allgemeiner: der Bedeutungsuibertragung) explizieren, daß die Carnapsche Konzeption von L-Relationen und Relationen in einzelnen möglichen Welten sinnvoll ist.

Sie wird allerdings im Falle der Metapher - zum Unterschied von anderen Fällen der Bedeutungsübertragung wie Allegorie, bestimmten Fällen von Symbolik und Gleichnis - erweitert werden müssen um das Konzept der Ordnung der Bedeutungskomponenten, die einem Lexem zugeordnet werden können. Wir können von diesem Erklärungsweg die oben diskutierte Möglichkeit D ausschließen, da keine metaphorische Interpretation, ja überhaupt keine sinnvolle Interpretation von (3) möglich ist - hier liegen einfach verschiedene Sprachen vor (d. h. Junggeselle bzw. verheiratet bedeuten einfach nicht das, was sie im Deutschen heute bedeuten). Folgerungen aus diesem Zustand sind natürlich müßig.

Beim Versuch, (3) Sinnvollheit und damit eine Interpretation zuzuteilen, wird offenbar von den "Normalbedeutungen" (vgl. Seite 94) von verheiratet und Junggeselle ausgegangen. Dabei werden Bedeutungspostulate wie (2') fallengelassen; die Bedeutungsanalysen der beiden Wörter (die Eigenschaften oder "Intensionen“, wie Carnap sagen würde) ändern sich nicht hinsichtlich der Menge der zuteilbaren Bedeutungsmerkmale, sondern hinsichtlich ihre Priorisierung untereinander (siehe das Kapitel „Priorisierung von Merkmalen“). Für das Beispiel (1) hat etwa folgende Umpriorisierung stattzufinden:

(5) JUNGGESELLE 1 : (Mann $) \wedge($ Erwachsen $) \wedge \neg$ (Verheiratet $) \wedge \ldots$ $\wedge\{($ Allein $) \wedge \ldots \wedge$ (Bindung an eine Frau alleine $) \wedge$

$\wedge$ (Häuslichkeit $) \wedge \ldots\}$ 
(6) JUNGGESELLE 3 : (Mann $) \wedge($ Erwachsen $) \wedge($ Allein $) \wedge($ Häuslichkeit $) \wedge$ $\wedge$ (Bindung an eine Frau alleine) $\wedge \ldots$

[Der Index unter dem Lexem in Blockbuchstaben zeigt die Welt an, für welche die Bedeutungsanalyse gilt: "1" also für die Welt, in der (1) notwendig wahr ist, „3" für die Welt, in $\operatorname{der}(3)$ nicht widersprüchlich ist.]

Nach der Intension von Junggeselle ist das Postulat (2) bzw. (2') analytisch, nach der Carnapschen Terminologie L-wahr. Würden wir die Extension von JunggeselLe in $\mathrm{W}_{1}$ absuchen, so würden wir auf keinen Menschen stoßen, auf den die Prädikate (Junggeselle) und (Verheiratet) gleichermaßen zutreffen. Wechseln wir hingegen die Welt, so daß (3) sinnvoll ist, so muß sich die Intension von JungGESELLE derart ändern, daß (Verheiratet) und (Junggeselle) kompatibel werden, genauer: daß Junggeselle das Prädikat (Verheiratet) zugeteilt werden kann. Das geschieht durch die andere Merkmalpriorisierung in JungGESELLE $_{3}$ - und dies entspricht offenbar genau unserer Intuition. In (3) ist für Junggeselle die Eigenschaft (Nichtverheiratet) nicht mehr vorrangig, so daß (2) nicht mehr gilt. Mit anderen Worten: Hat dieses Konzept der Priorisierung von Eigenschaften eine Entsprechung in kognitiven Ablauf, so sind Bedeutungspostulate in irgendeiner Weise abhängig von der Priorität, mit denen sie in einer Bedeutungsanalyse der Art oben [(5) und (6)] auftauchen. Wenn wir solche Merkmale (im technischen Sinne) auffassen als Eigenschaften, die einem Namen (Lexem) zugesprochen werden können müssen, um die Verwendung dieses Namens (Lexems) zutreffend zu machen, dann folgt aus dem Vorhergehenden, daß die Relevanz dieser Merkmale in einer bestimmten Zustandsbeschreibung mit zunehmender links-rechts-Reihung als Verifikationsbedingungen für das Lexem abnimmt. Bedeutungspostulate der Carnapschen Konzeption sind jeweils Aussagen über hochpriorisierte Eigenschaften der prädizierten Lexeme. Für die Metapher ist noch anzufügen, daß trotz der Aufgabe von (2) in der Welt, die (3) Sinnvollheit zuordnet, der Bezug auf die L-Wahrheit von (2) bestehen bleibt. D. h. erst das Bewußtsein darüber, daß man ein bestehendes analytisches System aufgibt, um Sinnvollheit für eine Prädikation zu erreichen, garantiert den Metaphernstatus. Dies drücke ich im folgenden durch die Wie-Relation aus (Abschnitt 5.). Ist dieses Bewußtsein nicht mehr vorhanden (sprachhistorisch: gehen solche Bedeutungspostulate durch andere Verwendungen des einen oder anderen Lexems verloren; genetisch: sind solche Postulate noch nicht aufgestellt worden), 
dann handelt es sich einfach um Bedeutungsänderungen, um "neue" Bedeutungen, jedoch nicht mehr um übertragene Bedeutungen. Ưbertragene Bedeutungen setzen den durch die Wie-Relation formulierten Bezug auf L-Wahrheit oder L-Falschheit im Carnapschen Sinne voraus. Noch eine Bemerkung zu der oben angedeuteten Unterscheidung zwischen Metaphern und anderen Typen der Bedeutungsübertragung auf der Grundlage dieser Überlegungen. Es scheint, daß für Metaphern gilt, daß Bedeutungspostulate [wie (2) bzw. (2')] aufgegeben werden, ohne durch neue (eventuell widersprechende) ersetzt $\mathrm{zu}$ werden. Konkret heißt das, daß der Bezug zur Normalbedeutung der Lexeme jeweils wiederhergestellt wird, sobald der Kompatibilisierungsprozeß abgelaufen ist. Bei der Allegorie und über einen längeren Textabschnitt konsistenten (d. h. in einer bestimmten Welt konsistenten) Symbolik („Textmetapher", vgl. Anm. 2) bleibt jedoch die neue alternative Welt (konstituiert mithilfe der die Metapher sinnvoll machen Bedeutungspostulate) über eine bestimmte Textstrecke hin aufrechterhalten.

\section{Zur Tiefenstruktur von Metaphern mithilfe von Analogieregeln}

Die einzig formal expliziten Beobachtungen zur Metaphernproduktion und -interpretation hat Kintsch (1972) angestellt. Ich folge ihnen grundsätzlich, aber nicht im Detail und versuche, ihre Anwendbarkeit an weiteren Beispielen zu verfolgen. Die grundlegende Annahme von Kintsch ist die, daß Metapher durch Analogieregeln, die quasi-prädikatenlogisches Format haben, erklärt werden. Diese Annahme ist gut begründet: Es hat sich in der Linguistik die Erkenntnis eingestellt, daß ein - eventuell etwas modifizierter - Prädikaten- und Relationenkalkül eine semantisch adäquate Beschreibung von Satzstrukturen liefert; speziell für das Verständnis des Status semantischer Merkmale für lexikalische Elemente ist dies von Weinreich (1966), Bierwisch (1969), Abraham (1972a) u. a. verfolgt worden. Unabhängig davon gibt es psychologische Beobachtungen, die wahrscheinlich machen, daß die Organisation des Denkens der Prädikatenlogik ähnlich ist (Wygotsky 1962). Letztlich bedient sich auch die Computerlinguistik in der Simulation natürlicher durch Sprachen formal-logischer Kalküle.

Es gehören über die Sätze mit hochgradiger Akzeptabilität hinaus noch zusätzliche Regeln, um Metaphern in der systematischen Beschreibung interpretierbar, rezipierbar und produzierbar zu machen. 
(1) Der Regen trommelt auf das Dach. ${ }^{5}$

Die Regelverletzung ist abzulesen aus der Charakteristik des Lexikoneintrags: TrommeL- $[+[+$ Agens $]-\ldots]$

wobei gilt: (Agens) $\neq$ (Verursacher), wohl aber (Agens) $\supset$ (Verursacher).

Bei der Metaphernproduktion und Metapherninterpretation - besser: beim Versuch sich eine Nichtakzeptabilität als Metapher zu erklären, d. h. nicht sofort als nichtinterpretierbar abzuweisen - kann nun folgende Kette von Bedeutungstransfers aufgebaut werden ${ }^{6}$ :

(2) Wenn der Regen kurz und periodisch auf das Dach schlägt, dann produziert dies dumpfe Töne so wie jemand, der auf eine Trommel schlägt

(三 der Trommelt)

(Diese Satzreihe gehört zu den direkt akzeptablen Propositionen.) [Metapher „in absentia“, d. h. es ist in der Oberfläche der Metapher der Substituent nicht vorhanden]. Nur eine Analogie ermöglicht - das ist die These - das Einsetzen von Trommeln anstelle von schläGT KURZ UND PERIODISCH in (2). Eine Reihe von möglichen Formen der Analogiebildung lassen sich auf Formen des klassischen Schließens zurückführen, für den Fall von (1) auf folgende Weise:

1. Prämisse: „es gilt" (Jemand schlägt auf die Trommel) $\supset$ (Dies erzeugt dumpfe Töne)

2. Prämisse: "nun aber": (Der Regen trommelt auf das Dach) $\supset$ (Dies erzeugt dumpfe Töne)

bzw.

(Der Regen schlägt kurz und periodisch auf das Dach auf) $\supset$ (Dies erzeugt dumpfe Töne)

(3) „daher" wiE [(Jemand schlägt auf die Trommel', (Der Regen trommelt auf das Dach)]

Das heißt aber mit anderen Worten auch, (2) ist die Bedeutung von (1). Allgemein ist die Arbeitsthese: Nur wenn einem Satz mit geringer oder niedriggradiger Akzeptabilität eine Analogiestruktur vom Typus in (3) zugeordnet werden kann, ist dieser Satz interpretierbar. Ob andere,

5 Ein Großteil der Beispiele in diesem Abschnitt ist von Kintsch 1972 übernommen.

"Hier im besonderen weicht meine "Tiefenstruktur" der Metapher von der Erklärung durch Kintsch ab, der ohne Konditional formuliert. Dadurch entstehen nicht nur eine Reihe von nichtakzeptablen Formulierungen im Prädikatenkalkül (Vermengung von extensionaler mit intensionaler Argumentation), sondern es kommt auch nicht zu der m. E. wesentlichen Form der Analogiebildung mithilfe der Regeln des logischen Schließens. 
nichtmetaphorische Oberflächenstrukturen durch Analogie wie in (2) zu erklären sind, muß dahingestellt bleiben. Solange also empirisch keine Annahme vertretbar erscheint, die dieses Kriterium wertlos macht, möchte ich davon ausgehen, daß mit dies ein Analogietypus die "Tiefenstruktur" einer Metapher beschrieben ist.

Für die folgenden beiden Beispiele schreibe ich nicht die Schlußregel explizit auf, sondern nur jeweils die expliziten Analogiestrukturen [" " vor einem Satz bedeutet niedriggradige Akzeptabilität, „, “ bedeutet hochgradige Akzeptabilität]:

Der Nebel kommt auf den Pfo- $\equiv+{ }^{+}$Der Nebel kommt und dies ten einer kleinen Katze geschieht so weich und unbörbar, wie wenn eine Katze auf ibren Pfoten kommt

Der Nebel büllt die Brücke ein $\equiv+$ Der Nebel büllt die Brücke wie ein nasses Tuch ein und dies macht sie naß und unsichtbar, wie wenn ein nasses Tuch sie einbüllte.

[Metapher in "praesentia", das heißt Substitut und Substituent sind in der Oberflächenstruktur gleichermaßen vorhanden].

Da wir in der komprimierten Form der letzten Analogie einiges unterdrücken, sei sie noch einmal etwas expliziter angeschrieben.

(4) Der Nebel umbïllte die Brücke wie ein nasses Tuch $\equiv$ Was von einem nassen Tuch oder Nebel umhüllt wird, wird naß (三 Wenn etwas von einem Tuch oder Nebel umhüllt wird, dann wird es naß) $\wedge$ Die Brücke wird vom Nebel umhüllt

Dies läßt sich folgendermaßen im Prädikatenkalkül niederlegen:

(5) $[$ UMHÜLlt $(x, n T) . \supset$.NASS $(x) \wedge$ UNSICHTBAR $(x)] \wedge$ [uMHÜLLT $(\mathrm{B}, \mathrm{N})]$

wobei: nT ... nasses Tuch, B ... Brücke, N ... Nebel

Das die Analogie erklärende Schlußschema lautet folgendermaßen:

UMHÜLLT $[\mathrm{x}, \mathrm{nT}$ v $\mathrm{N}]$. $\supset$.NASS $(\mathrm{x}) \wedge$ UNSICHTBAR $(\mathrm{x})$

Uмнӥ̈LLT [B, N]

NASS $(B) \wedge$ UNSICHTBAR $(B)$

Nur kurz möchte ich bei diesen Beispielen noch einmal eingehen auf die Notwendigkeit der Umordnung der semantischen Merkmale bei der Erklärung der metaphorischen Funktion eines bestimmten lexikalischen Elements. Bei der metaphorischen Verwendung von trommeln in dem Kontext der Regen trommelt handelt es sich um eine Generalisierung 
der Bedeutung insofern, als die hochgradig akzeptable Einschränkung mithilfe des Merkmals [+ Menschlich] aufgehoben wird. Betrachten wir zu diesem Argument der Priorisierung aber noch ein anderes Beispiel:

(6) Flußbett $=-$ Bett des Flusses

BETT $=$ A: (zum Liegen $) \wedge($ zum Schlafen $) \wedge($ für den Menschen $) \vee$ (für Tiere) $\wedge \ldots \wedge$ B: (damit man gegen unbeabsichtigte Bewegungen in der vorgesehenen Position bleibt) $\wedge \ldots$

(wobei mit A Anwendungsbedingungen indiziert werden, die für das Lexem Bett vorrangiger sind als die mit B indizierten).

Nun aber: $\mathrm{X}$ des Flusses $=($ damit Wasser in der vorgesehenen Position bleibt)

daher: $\mathrm{X}=$ Bett des Flusses

Das letzte Beispiel der Schlußfolgerung übrigens prozedualisiert die Metaphern produktion. Man könnte versuchen, sich die Metaphernproduktion vorzustellen als Prozesse, die nachrangige Anwendungsbedingungen wie die unter $\mathrm{B}$ oben primär postulieren, um damit $\mathrm{zu}$ lexikalischen Elementen in metaphorischer Funktion zu kommen. Die Metaphern rezeption greift diesen Prozeß offenbar am anderen Ende an: Wird jemand mit einer lexikalischen Inkompatibilität konfrontiert, so liegt genau das X des letzten Schlußschemas vor, und der Metapherninterpretator hat nun jene Anwendungsbedingungen (Implikate) zu finden, die sowohl für das Substituens wie auch das Substituendum gleichermaßen gelten. Nach diesen Überlegungen also teile ich die folgenden expliziteren Formulierungen ein in solche der Metaphern produktion und der Metaphern rezeption.

\subsection{Analogieregeln zur Metaphernproduktion.}

Die Analyse der Metaphernproduktion sei an dem Beispiel Regen trommelt auf das Dach aufgehängt.

(7) $\mathrm{Q}=\mathrm{R}(\mathrm{a}, \mathrm{b}) \supset \mathrm{S}(\mathrm{c}, \mathrm{d})$

„wenn Regen auf das Dach fällt,

(8) $Q^{\prime}=T(e, f) \supset S(c, d)$ entsteht ein dumpfer Ton"

(9) $P^{*}=\operatorname{WIE}\left[\mathrm{Q}, \mathrm{Q}^{\prime}\right]$ "wenn jemand trommelt, entsteht ein dumpfer Ton" „wenn Regen auf das Dach fällt, ist es, wie wenn jemand trommelt"

(Nur der Einfachheit halber werden hier die Propositionsstrukturen mit jeweils zwei Argumenten dargestellt.) Der Such- und Erklärungs- 
vorgang bei der Metaphernproduktion geht aus von $R$ und führt über $S$ zu T. Prüfinstanz ist dabei $P *$. Dabei gilt für $P^{*}$ :

(10) $\mathrm{P}^{*}=\{\mathrm{x} \mid \mathrm{x} \supset \mathrm{S}(\mathrm{c}, \mathrm{d})\}$

$$
\begin{aligned}
& T(e, f) \in P^{*} \\
& R(a, b) \in P^{*}
\end{aligned}
$$

(10) bringt zum Ausdruck, daß es eine Äquivalenzklasse P* derart gibt, daß sämtliche Elemente dieser Klasse (Propositionen der Form T, R) die Bedingung „ $\mathrm{S}(\mathrm{c}, \mathrm{d})$ " erfüllen. Es ist deutlich, daß es in dieser Klasse nicht nur ein einziges Element geben muß, d. h. daß es für eine bestimmte Vorstellung nicht eine einzige metaphorische Realisierung zu geben braucht. Im besonderen wird durch die Art der Hypothesen über die Elemente dieser Klasse, hier konkret $\mathrm{T}$ und $\mathrm{R}$, eine $\mathrm{Grad}$ skala von Äquivalenzen festgelegt. So kann es unter Umständen niedrigergradige und höhergradige Äquivalenzen im Hinblick auf die dann-Folgerung $S(c, d)$ in Abhängigkeit davon geben, wieviele der Hypothesen über die Richtigkeit von $\mathrm{R} \supset \mathrm{S}$ und $\mathrm{T} \supset \mathrm{S}$ übereinstimmen. Dies ist das Problem der Priorität der Merkmalsanalyse, sowie es in Abschnitt 4 diskutiert wurde.

Wenn die durch das WIE-Prädikat ausgedrückte Analogie P* die Tiefenstruktur einer Metapher ist, dann bleibt die Frage, wie wir aus dieser Tiefenstruktur zur Oberflächenstruktur der Metapher gelangen. Dies geschieht offenbar (und hier schließe ich mich wieder der Darstellung von Kintsch an) mithilfe von bestimmten Typen von Verschmelzungsregeln (VR). Im ersten Schritt werden dabei Q und Q' um das gemeinsame Implikat reduziert:

(11) $\mathrm{Q}=+\mathrm{R}(\mathrm{a}, \mathrm{b})$

$$
Q^{\prime}={ }^{+} T(e, f)
$$

$\Rightarrow \operatorname{WIE}[\mathrm{T}(e, f), R(a, b)]$

Der WIE-Vergleich ist dann reduziert auf:

(12) WIE(Regen fällt auf das Dach, jemand trommelt)

Nun kann der Fall eintreten, daß in Q und Q' die Prädikate identisch sind:

(13)

$$
\begin{aligned}
& Q^{\prime}=R(a, b) \\
& Q^{\prime}=R(e, f) \\
& P^{*}=\operatorname{Vir}[R(a, b), R(e, f)]
\end{aligned}
$$

Die erste Verschmelzungsregel $\left(\mathrm{VR}_{1}\right)$ wird so anzusetzen sein:

(14) $\operatorname{VR}_{1}: P=\operatorname{WiE}[R[(a, b),(e, f)]]$

Dieser Regel wird offenbar wirksam bei der Metapher der Nebelkommt auf den Pfoten einer kleinen Katze, wo nämlich den Argumenten $\mathrm{Ne}$ - 
bel genau so wie Katze das Prädikat kommt auf weichen Pfoten gemeinsam ist. Dieser Verschmelzungsprozeß hat zweifellos eine Reihe von Untertypen, die allesamt vergleichbar sind mit dem in der Linguistik seit Ross bekannten Vorgang des "gapping“.

Ein anderer Typus von Verschmelzung liegt vor im Falle von verschiedenen Verben:

(15) $Q=R(a, b)$

$Q^{\prime}=T(e, f)$

$\Rightarrow P^{*}=\mathbb{W I E}[R(a, b), T(e, f)]$

(16) $\operatorname{VR}_{2}: P=\operatorname{WIE}[T[(a, b),(e, f)]]$

$\mathrm{VR}_{2}$ ist wirksam in der Trommel-Metapher: sowohl Regen wie auch Trommler haben das Prädikat trommeln.

Um zwischen der Metapher „in absentia“ und „in praesentia“ zu trennen, muß offenbar noch eine fakultative $\mathrm{VR}_{3}$ hinzugefügt werden, in der nämlich der explizite WIE-Vergleich getilgt wird:

(17) $\mathrm{VR}_{3}: \mathrm{P}=\mathrm{T}[\mathrm{a}, \mathrm{b}]$

Die Anwendung dieser Regel würde aus der Metapher Der Nebel bïllte die Brücke ein wie ein nasses Tuch machen Der Nebel büllte die Brücke (naß) ein, das heißt den expliziten WIE-Vergleich wie ein nasses Tuch tilgen. $\mathrm{Zu}$ diesen Verschmelzungsregeln kommen eine Reihe typischer Regeln, die einen Wechsel zwischen Tiefenkasus beschreiben: $z$. B. die Personifikationsregeln $(\mathrm{O} \Rightarrow \mathrm{A})$ :

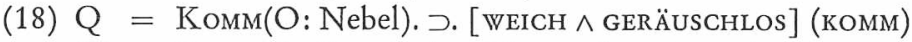

$\mathrm{Q}^{\prime}=\operatorname{Komm}(\mathrm{A}, \mathrm{I}$ : auf den Pfoten einer Katze). $\supset[$ weIcH $\wedge$ GERÄUSCHLOS] (KOMM)

$\mathrm{P}^{*}=\operatorname{wit}[\operatorname{Komm}(\mathrm{O}), \operatorname{Komm}(\mathrm{A}, \mathrm{I})]$

$\mathrm{P}=\operatorname{Komm}(\mathrm{O}, \mathrm{I})$

\subsection{Analogieregeln zur Metaphernrezeption}

Das Schlußschema bei der Rezeption (Interpretation) einer Metapher, wo das Substituens nicht, wohl aber das Substituendum bekannt ist, sind identisch mit denen der Metaphernproduktion, nur sind eben Schlußfolgerung und erste Prämisse vertauscht.

Daß Metaphern schwieriger zu rezipieren (manchmal überhaupt nicht als Metaphern zu erkennen) sind als zu produzieren, liegt offenbar daran, daß die niedriggradig akzeptable Metapher eine sehr große Menge von Hypothesen erlaubt, aus denen verschiedene Implikate abgeleitet werden können, die wiederum verschiedene Äquivalenzklassen konstituieren. Aus diesen verschiedenen Äquivalenzklassen wie- 
derum sind verschiedene Implikatoren, d. h. Substituentes (normale lexikalische Verwendungen) zu erschließen. Die Reihe der Möglichkeiten wird noch vergrößert dadurch, daß erstens nicht bekannt ist, wie gleichrangig die Äquivalenzklassen sind, und zum zweiten, welches die normalen Hypothesen (die normalen Bedeutungen, jene alternative Welt, die der Konstitution der Normalbedeutung zugrundegelegen hat) des Metaphernproduzenten waren.

-(schwarze -(Milch: der Frühe))

lexikalische Hypothesen...$\cdots \ldots\left\{\begin{array}{l}\uparrow \\ \text { (weiß) } \\ \text { (ohne Farbgliederung) }\end{array}\right.$

enzyklopädische Hypothesen $\ldots . .\left\{\begin{array}{l}\text { (Helligkeit) } \\ \text { (Sichtbarkeit oder } \\ \text { Preisgabe alles Wahrnehm- } \\ \text { baren) }\end{array}\right.$

-(schwarze: Milch der Frühe)

enzyklopädische Hypothesen ...... $\left\{\begin{array}{l}\begin{array}{l}\uparrow \\ \text { (unbekannt) } \\ \text { (traurig) } \\ \text { (gefährlich) } \\ \text { (todbringend) }\end{array}\end{array}\right.$

Die niedriggradige Akzeptabilität dieser komplexen Metapher liegt an zwei Stellen („: “ kennzeichnet die Inkompatibilitätsstellen): Durch Zuordnung von Hypothesen über den (möglichen) Sinn von Milch und schwarz entstehen kompatible (interpretierbare) Strukturen. Die als Hypothesen bezeichneten Merkmale vertreten, in die oben dargestellte Sprache der Analogiebildung übertragen, sowohl Implikate (S) wie auch die Substituentes (R).

\section{Bibliograpbie}

Abraham, W. (1971): Stil, Pragmatik und Abweichungsgrammatik. In: A. von Stechow: Beiträge zur generativen Grammatik. Braunschweig 1971. S. 1-13.

- (1972): The necessity of inserting „speaker" and "hearer" as basic categories of a practicable grammatical model. In: Corder / Roulet (Hrsg.), 31-46.

Abraham, W. (1972a). Komponentialanalyse als Ubersetzungssprache in einem Dokumentenretrieval. In: H. Preiß (Hrsg.) Praktische Fälle über Aufbau und Funktionsweise betrieblicher Teilinformationssysteme. München 1972, $56-112$.

- (1973): Einige formale Eigenschaften von Komponentialanalysen. In: A. P. ten Cate / P. Jordens (Hrsg.): Linguistische Perspektiven. Tübingen. 
Abraham, W. (1975): A linguistic Approach to metaphor. In: W. Abraham (Hrsg.) Ut Videam. Contributions to an Understanding of Linguistics. Lisse.

Abraham, W. / K. Braunmüller (1971): Stil, Metapher und Pragmatik. In: Lingua 28 , no. 1, 2. S. 1-48.

Apostel, L. (1971): Further remarks on the pragmatics of natural languages. In: Bar-Hillel (Hrsg.) Pragmatics of Natural Languages. Dordrecht, 1-34.

Asch, S. / H. Nerlove (1960): The Development of Double-Function Terms. In: B. Kaplan / S. Wapner: Perspectives in Psychological Theory. New York.

Austin, J. (1962): How to do Things with Words. Oxford.

Bartsch, R. (1971): Semantische Darstellung von Prädikaten. In: Linguistische Berichte 13, 33-42.

Bickerton, D. (1969): Prolegomena to a Linguistic Theory of Metaphor. In: Foundations of Language 4. S. 34-52.

Bierwisch, M. (1969): On certain problems of semantic representation. In: Foundations of Language 5, S. 153-184.

Bierwisch, M. / Kiefer, F. (1969): Remarks on Definitions in Natural Language. In: Kiefer (ed.) 1969: Studies in Syntax and Semantics. Dordrecht, $55-79$.

Black, M. (1962): Models and Metaphors. Ithaca, N. Y.

Carnap, R. (1958): Meaning and Necessity. Phoenix.

Cohen, L. J. (1971): Some Remarks on Grice's views about the logical Particles of Natural Language. In: Y. Bar-Hillel (Hrsg.) Pragmatics of Natural Languages. Dordrecht, 50-68.

Cohen, L. J. / Margalit A. (1970): The Role of Inductive Reasoning in the Interpretation of Metaphor. In: Synthese 21, 469-487.

Corder, P. / Roulet, E. (1972): Theoretical Linguistic Models in Applied Linguistics. Brüssel.

van Fraassen, B. C. (1971): Formal Semantics and Logic. New York.

Goodman, N. (1968): Languages of Art: An Approach to the Theory of Symbols. Indianapolis.

Helmer, J. (1972): Metaphor. In: Linguistics 88, 5-14.

Jakobson, R. (1960): Concluding Statement: Linguistics and Poetics. In: T. Sebeok (ed.) Style in Language. New York.

Jakobson, R. / M. Halle (1956): Fundamentals of Language. The Hague.

Kamlah, W. / Lorenzen, P. (1967): Logische Propädeutik. B. I. Hochschultaschenbücher.

Kintsch, W. (1971): Notes on the semantic structure of memory. Studies in Mathematical Learning Theory and Psycholinguistics. CLIPR Publication No. 4. University of Colorado, Computer Laboratory for Instruction in Psychological Research.

- (1972): Notes on the structure of semantic memory. In: E. Tulving / W. Donaldson (eds.): Organization of Memory. New York/London.

Matthews, R. J. (1971): Concerning a ,Linguistic Theory' of Metaphor. In: Foundations of Language, 3. 413-426. 
Osgood, C. (1960): Some Effects of Motivation on Style in Encoding. In: Sebeok (ed.) Style in Language. New York.

Richards, I. A. (1936): The Philosophy of Rhetoric. Oxford.

Siebert, H. J. (1973): Isotopie der Metapher. Hektogramm, Bielefeld.

Weinreich, U. (1966): Explorations in Semantic Theory. In: Currend Trends in Linguistics. University of Chicago. Chicago, Illinois. S. 240-251.

Wygotsky, L. S. (1962): Thought and Language. Cambridge/Mass. M. I. T. [dt. Denken und Sprache. 1964] 